\title{
Mode Transitions in Magnetically Shielded Hall Effect Thrusters
}

\author{
Michael J. Sekerak* Benjamin W. Longmier ${ }^{\dagger}$ and Alec D. Gallimore \\ University of Michigan, Ann Arbor, MI, 48105 \\ Wensheng Huang§ Hani Kamhawi $₫$ \\ National Aeronautics and Space Administration, Glenn Research Center, Cleveland, Ohio, 44135 \\ and \\ Richard R. Hofer ${ }^{\|}$Benjamin A. Jorns** and James E. Polk ${ }^{\dagger \dagger}$ \\ Jet Propulsion Laboratory, California Institute of Technology, Pasadena, CA 91109
}

\begin{abstract}
A mode transition study is conducted in magnetically shielded thrusters where the magnetic field magnitude is varied to induce mode transitions. Three different oscillatory modes are identified with the 20-kW NASA-300MS-2 and the 6-kW H6MS: Mode 1) global mode similar to unshielded thrusters at low magnetic fields, Mode 2) cathode oscillations at nominal magnetic fields, and Mode 3) combined spoke, cathode and breathing mode oscillations at high magnetic fields. Mode 1 exhibits large amplitude, low frequency $(1-10 \mathrm{kHz})$, breathing mode type oscillations where discharge current mean value and oscillation amplitude peak. The mean discharge current is minimized while thrust-to-power and anode efficiency are maximized in Mode 2, where higher frequency (50-90 kHz), low amplitude, cathode oscillations dominate. Thrust is maximized in Mode 3 and decreases by $5-6 \%$ with decreasing magnetic field strength. The presence or absence of spokes and strong cathode oscillations do not affect each other or discharge current. Similar to unshielded thrusters, mode transitions and plasma oscillations affect magnetically shielded thruster performance and should be characterized during system development.
\end{abstract}

\section{Nomenclature}

$B_{r} \quad$ Maximum radial magnetic field, $\mathrm{G}$

$B_{r}^{*} \quad$ Reference maximum radial magnetic field, G

$B_{r} / B_{r}^{*} \quad$ Normalized magnetic field

$f \quad$ Frequency, $\mathrm{kHz}$

$I_{D} \quad$ Discharge current, $\mathrm{A}$

$I_{I M} \quad$ Inner magnet coil current, $\mathrm{A}$

$I_{O M} \quad$ Outer magnet coil current, A

$j \quad$ Discharge current density, $\mathrm{mA} \mathrm{cm}^{-2}$

$m \quad$ Spoke order

\footnotetext{
*Post-Doctoral Research Fellow, Plasmadynamics and Electric Propulsion Laboratory, msekerak@umich.edu

$\dagger$ Assistant Professor, Plasmadynamics and Electric Propulsion Laboratory, longmier@umich.edu

¥Arthur F. Thurnau Professor and Director, Plasmadynamics and Electric Propulsion Laboratory, alec.gallimore@umich.edu

$\S$ Research Engineer, In-Space Propulsion Systems Branch, wensheng.huang@nasa.gov

IResearch Engineer, In-Space Propulsion Systems Branch, hani.kamhawi-1@nasa.gov

\| Senior Engineer, Electric Propulsion Group, richard.r.hofer@jpl.nasa.gov

**Associate Engineer, Electric Propulsion Group, benjamin.a.jorns@jpl.nasa.gov

${ }^{\dagger}$ Principal Engineer; Propulsion and Materials Engineering Section, james.e.polk@jpl.nasa.gov
} 


$\begin{array}{ll}m_{0} & \text { Spoke order } m=0 \text { or global light intensity } \\ \dot{m}_{a} & \text { Anode mass flow rate, } \mathrm{mg} \mathrm{s}^{-1} \\ P_{D} & \text { Discharge power, } \mathrm{kW} \\ T & \text { Thrust, } \mathrm{N} \\ T / P & \text { Thrust-to-power, } \mathrm{mN} \mathrm{kW}^{-1} \\ V_{D} & \text { Discharge voltage, } \mathrm{V} \\ \eta_{a} & \text { Anode efficiency }\end{array}$

\section{Introduction}

Hall Effect Thrusters (HETs) have been under development for over 50 years with significant experimental and flight histories. ${ }^{1}$ Mode transitions have been commonly observed throughout their development as noted by some of the early pioneering Russian research. ${ }^{2}$ HETs have several parameters that define a single operating point such as discharge voltage, magnetic field strength (or magnet coil current), anode mass flow rate and cathode mass flow rate. Mode transitions are generally described as the point while varying one parameter and maintaining all others constant, a sharp discontinuity is observed in the discharge current mean value and oscillation amplitude. A previous investigation using a 6 - $\mathrm{kW}$ laboratory HET called the H6 induced mode transitions by varying magnetic field magnitude where the changes in performance and plasma oscillations were examined with ultra-fast imaging and high-speed probes. ${ }^{3,4}$ The purpose of the present work is to show preliminary results from the first investigation of mode transitions in magnetically shielded thrusters.

Discharge channel erosion limiting thruster life has been a problem since the inception of HETs. Recent advancements in magnetic field design have potentially solved the issue of channel erosion through a technique known as magnetic shielding. ${ }^{5,6}$ Magnetic shielding of HETs was first observed in 2009 on a BPT-4000 during long-duration ground testing ${ }^{7}$ where the channel erosion decreased below the threshold of measurements after 5600 hours of operation. ${ }^{8}$ The physics behind the decreased erosion were investigated through simulations ${ }^{5,7}$ and experiments, ${ }^{6}$ and demonstrated on a modified H6 at the NASA Jet Propulsion Laboratory (JPL). The modified thruster, called the H6MS, reduced erosion below the threshold of detection by limiting plasma contact with the discharge channel walls. This has enabled the use of conductive discharge channel walls made from carbon ${ }^{9}$ without significant performance degradation.

In the present work, mode transitions in two magnetically shielded thrusters are investigated using the same techniques described in Refs. 3 and 4 for the investigation of unshielded thrusters. The two thrusters investigated are the NASA-300MS-2 tested at NASA Glenn Research Center(GRC) and the H6MS tested at NASA JPL. Both magnetically shielded thrusters exhibit two transition points with three different oscillatory modes.

\section{Mode Transition Background}

Previous researchers have identified mode transitions in unshielded HETs ${ }^{2,10,11}$ where a small change in a thruster operating parameter such as discharge voltage, magnetic field or mass flow rates cause the mean discharge current and oscillation amplitude to increase significantly. A recent investigation using the H6 studied mode transitions induced by varying the magnetic field intensity while holding all other operating parameters constant, ${ }^{3,4}$ with an example reproduced in Fig. 1 . Two modes were identified and described as global oscillation mode and local oscillation mode. In global mode, the entire discharge channel is oscillating in unison and azimuthal spokes are either absent or negligible, with discharge current oscillation amplitude (Root-Mean-Square or RMS) greater than $10-100 \%$ of the mean value. In local oscillation mode, perturbations in the discharge current density are seen to propagate in the $E \times B$ direction at $1500-2200 \mathrm{~m} / \mathrm{s}^{4,12}$ with clear spokes shown in a High-Speed Imaging Analysis (HIA) Power Spectral Density (PSD). Spokes are localized oscillations that are typically 10-20\% of the mean discharge current density value. The discharge current oscillation amplitude and mean values are significantly lower than global mode as shown by the top plot in Fig. 1. The transition between global mode and local mode occurred at higher relative B-field strengths for higher mass flow rate or higher discharge voltage. The thrust was approximately constant through the mode transition, but the thrust-to-power ratio $(T / P)$ and anode efficiency $\left(\eta_{a}\right)$ decreased significantly in global mode with the peak values typically occurring near the transition point. ${ }^{4}$ 


\section{A. High-Speed Imaging Analysis (HIA)}

This section describes the HIA techniques used for the previous H6 mode transition investigation as well as the present H6MS and NASA-300MS-2 investigations. High-speed imaging was acquired with a Photron SA5 FASTCAM and a Nikon ED AF Nikkor 80-200 mm lens at its maximum aperture. The SA5 is capable of up to $1,000,000$ frams-per-second (fps) with $128 \times 16$ pixel resolution, but was used at 87,500 fps with $256 \times 256$ pixel resolution. The camera was $6 \mathrm{~m}$ downstream from the thruster, located outside of the vacuum chamber with a view of the thruster through a viewport. The high-speed images are analyzed using McDonald technique detailed in Refs. 3, 4, and 13; a brief summary is provided here. The video is imported into MathWorks MatLab where each frame is a $256 \times 256$ matrix of light intensity values and the DC component is subtracted individually from each pixel. The discharge channel is isolated and divided into 180 two-degree bins. The pixels in each bin are averaged together generating a $180 \times 1$ vector of light intensity for each frame. A 2-D plot of all frames is the spoke surface where the ordinate is azimuthal location around the discharge channel in clock positions and the abscissa is time with each vertical column of values representing one frame of video, as shown in the bottom row of plots in Fig. 1. Adding all bins together yields the $m=0$ or $m_{0}$ mode and was first shown by Lobbia ${ }^{14}$ to linearly correlate with the discharge current. During the mode transition investigation of Ref. 3 the same strong, linear correlation was also observed and used as the basis for converting light intensity to discharge current density assuming local discharge current is linearly related to local light intensity. This assumption is discussed in Ref. 4 and supported by hybrid-direct kinetic simulations. ${ }^{15}$

The discharge current density plots (bottom row plots in Fig. 1) yield valuable information on plasma oscillations within the discharge channel by showing the time-resolved, azimuthal distribution of discharge current density. Vertical features represent extremes in discharge current density that occur everywhere in the channel simultaneously and are observed for $B_{r} / B_{r}^{*}=0.52$ and 0.61 in Fig. 1 . Diagonal features are perturbations in discharge current density that propagate azimuthally around the discharge channel. Lines from upper-left to lower-right are propagating counter-clockwise (CCW) around the discharge channel and lines from the lower-left to upper-right are propagating clockwise $(\mathrm{CW})$. The $E \times B$ direction in the $\mathrm{H} 6$ is $\mathrm{CCW}$ and all azimuthally propagating features are in that direction as evidenced by $B_{r} / B_{r}^{*}=1.00$ and 1.48 in Fig. 1.

A 2-D Fourier transform of the discharge current density surface shows the peak frequencies for each spoke order, $m$. These are referred to as HIA PSDs with examples in the middle row plots of Fig. 1. As described by McDonald in his original derivation, $m$ is analogous to number of wavelengths per channel circumference. ${ }^{13}$ Hence $m=0$ or $m_{0}$ is no wave in the channel (the entire channel is dark or bright), $m=1$ means one wave in the channel (one half bright, the other dark), $m=2$ is two waves per channel (two bright regions, two dark regions), etc. Harmonics are noted at frequencies higher than the dominant frequency. If a spoke order has a very strong peak, then other spoke orders often show smaller peaks at the same frequency, which are noted to be non-physical artifacts of the processing techniques. ${ }^{16}$ As discussed by McDonald, ${ }^{16}$ the absolute peak values for HIA PSDs should only be compared within a given parameter study with the same experimental setup because the 2-D Fourier transform values are sensitive to camera CCD quantum sensitivity, lens aperture size, shutter speed or exposure time, vacuum chamber viewport optical quality, operating condition and other post-processing variables. In this investigation, all setup and processing parameters were held constant for a given thruster.

\section{B. H6 Mode Transition Results}

The normalized magnetic field, $B_{r} / B_{r}^{*}$, was varied by changing the inner and outer magnet coil currents in a constant ratio with all other parameters held constant including flow rates, discharge voltage, and chamber pressure. Maintaining a constant ratio of inner to outer coil current allowed the magnetic field magnitude to be varied without changing the shape. The quantity $B_{r} / B_{r}^{*}$ is the maximum radial magnetic field value at a particular setting of inner magnet current $\left(I_{I M}\right)$ and outer magnet current $\left(I_{O M}\right)$ divided by the reference maximum radial magnetic field $\left(B_{r}^{*}\right)$ at $I_{I M}=3.50 \mathrm{~A}$ and $I_{O M}=3.13 \mathrm{~A}$. The reference value is fixed across all operating conditions. Decreasing $B_{r} / B_{r}^{*}$ below a certain threshold was shown to repeatedly induce a mode transition where the mean discharge current increased and the discharge current oscillation amplitude (RMS) increased. An example of this transition is shown in the top plot of Fig. 1. The mean discharge current and oscillation amplitude are lowest in local mode $\left(B_{r} / B_{r}^{*}>0.61\right)$ and then increase sharply in global mode $\left(B_{r} / B_{r}^{*}<0.61\right)$. The transition point is $B_{r} /\left.B_{r}^{*}\right|_{\text {trans }}=0.61$. A defined transition $B_{r} / B_{r}^{*}$ is 
misleading because there is a transition region where the plasma exhibits both types of oscillations as shown in Fig. 1, however the transition typically occurred over only $\sim 10 \%$ change in $B_{r} / B_{r}^{*}$. Figures 12,13 and 14 of Ref. 3 shows that $B_{r} /\left.B_{r}^{*}\right|_{\text {trans }}$ increased with increasing flow rate and discharge voltage. The spokes shown in Fig. 1 for $B_{r} / B_{r}^{*}=1.00$ and 1.48 are localized oscillations that are typically $10-20 \%$ of the mean discharge current density value, while the oscillations in global mode $B_{r} / B_{r}^{*}=0.52$ can be $100 \%$ of the mean value. Refs. 3 and 4 conclude that the $\mathrm{H} 6$ and likely any similar thruster should be operated in local oscillation mode to minimize discharge current and maximize performance.
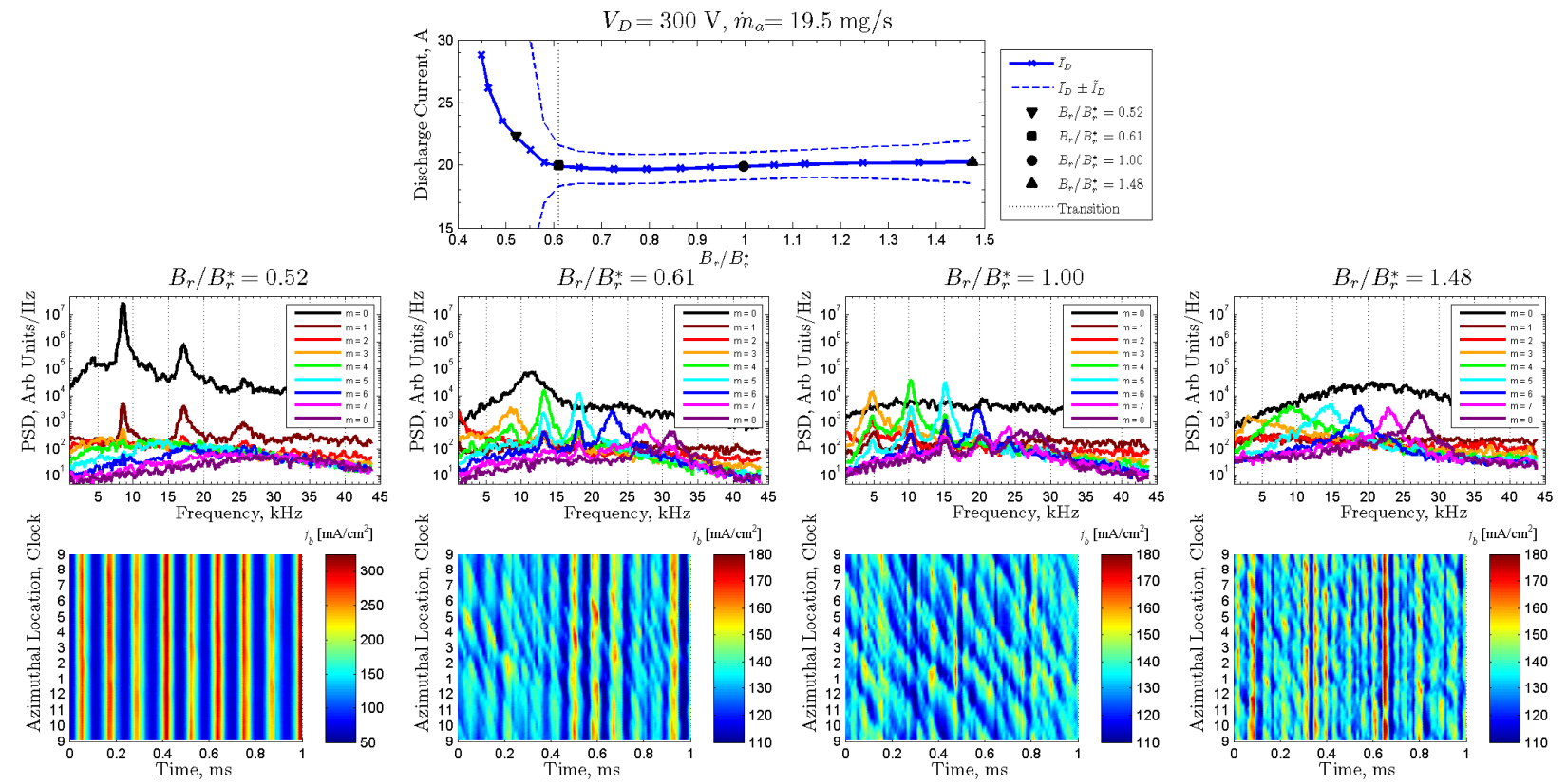

Figure 1. B-field sweep for $300 \mathrm{~V}, 19.5 \mathrm{mg} / \mathrm{s}$ showing transition at $B_{r} / B_{r}^{*}=0.61$ for the unshielded H6. The discharge current mean and oscillation amplitude are shown with the transition and for $B_{r} / B_{r}^{*}$ settings selected for further analysis. The middle row plots are HIA PSDs and the bottom row plots are discharge current density contour plots. The scale range for $B_{r} / B_{r}^{*}=0.52$ discharge current density is larger due to the magnitude of oscillations. A 500-Hz moving average filter has been applied to smooth all HIA PSDs. Reproduced from Figure 17 of Ref. 3.

\section{Experimental Setup}

\section{A. Magnetically Shielded 300M (NASA-300MS and NASA-300MS-2)}

\section{NASA-300MS and NASA-300MS-2 Information}

NASA is developing a 10-15 kW HET for a Solar Electric Propulsion Technology Demonstration Mission. As part of this effort, the NASA-300M thruster was modified to incorporate magnetic shielding and called the NASA-300MS, shown in Figure 2. For brevity, the NASA indicator in front of the thruster name will be dropped. The 300M is a magnetic layer HET with a centrally mounted cathode and a magnetic lens topology designed for high specific impulse missions. ${ }^{17}$ The magnetic circuit and the discharge channel walls of the $300 \mathrm{M}$ were modified to implement the magnetic shielding, but the rest of the thruster including the mean channel diameter remains unchanged. A second configuration of the 300MS was used with a shortened discharge channel length. The anode was moved down stream reducing the discharge channel length by $20 \%$ and is denoted as the $300 \mathrm{MS}-2 .^{18}$

Ref. 18 details an extensive effort at NASA GRC to characterize the 300MS with far field diagnostics including a Faraday probe, $E \times B$ probe, retarding potential analyzer, Langmuir probe, and thrust stand. The investigation concluded "the 300MS data shows $\sim 2 \%$ higher voltage utilization efficiency, $\sim 2.5 \%$ lower divergence efficiency, and $\sim 1.5 \%$ higher current utilization efficiency than the 300M data. The two thrusters have roughly the same values in charge and mass utilization efficiencies. The general conclusion is that the 
$300 \mathrm{M}$ and 300MS have very similar performance as measured by the anode efficiency." The 300MS and the 300MS-2 had the same performance within measurement uncertainty. ${ }^{18}$

All results presented here for mode transitions identified using HIA were performed on the 300MS-2. The magnetic field was directed radially outward so the $E \times B$ direction was CCW like the H6. The thruster was operated on xenon with an anode mass flow rate of $40 \mathrm{mg} / \mathrm{s}$ and cathode mass flow rate of $3.2 \mathrm{mg} / \mathrm{s}$ for an $8 \%$ Cathode Flow Fraction $(\mathrm{CFF})$. Two operating conditions were tested:

1. $V_{D}=300 \mathrm{~V}, I_{D}=50 \mathrm{~A}, P_{D}=15 \mathrm{~kW}$

2. $V_{D}=400 \mathrm{~V}, I_{D}=50 \mathrm{~A}, P_{D}=20 \mathrm{~kW}$

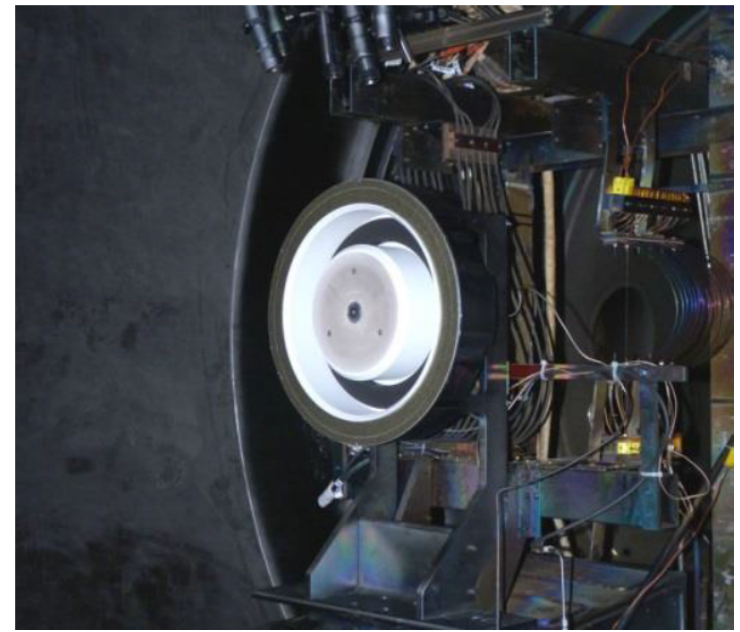

(a) On the thrust stand at NASA GRC. Reproduced from Figure 2 of Ref. 18

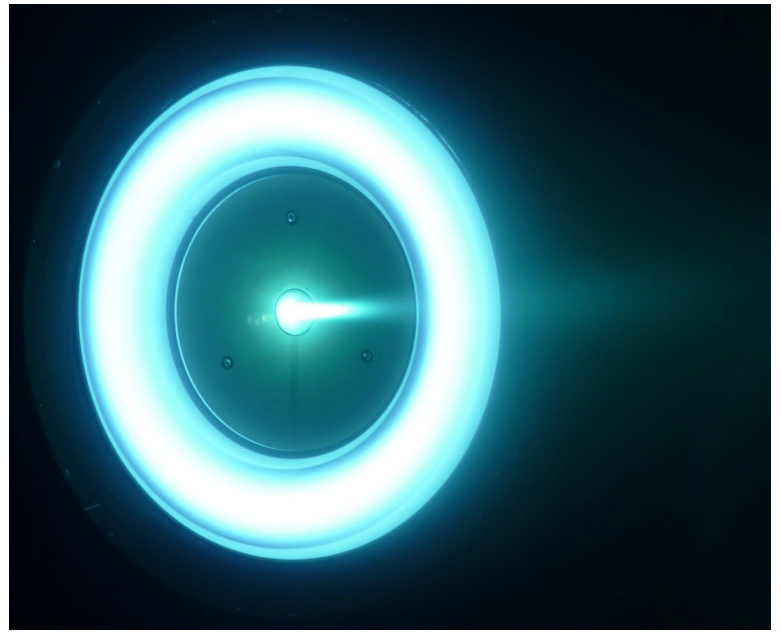

(b) Operating in VF-5 at $15 \mathrm{~kW}$.

Figure 2. The NASA-300MS at NASA GRC.

The 300M and 300MS both have independent inner and outer electromagnets that establish the magnetic field through interaction with the magnetic circuit. The same technique of varying magnetic field strength without varying shape used in the $\mathrm{H} 6$ investigation ${ }^{3,4}$ was employed here by maintaining a constant magnet coil current ratio of $I_{I M} / I_{O M}=1.05 \pm 0.01$. Note that no magnet field simulations or measurements were conducted at the different $I_{M}$ settings to verify the magnetic field shape was unaltered. This shortcoming is significant as the magnetic circuit was likely saturated at the highest magnet currents with the implications discussed in Section V.A.

\section{Experimental Setup at NASA GRC}

The tests were conducted in Vacuum Facility 5 (VF-5) at NASA GRC and are described in Ref. 18, with key metrics summarized here. VF-5 is a cylindrical vacuum chamber $4.6 \mathrm{~m}$ in diameter and $18.3 \mathrm{~m}$ long, and is equipped with cryo-panels and 20 oil diffusion pumps. The pressure measured by an ion gauge mounted near the thrust stand was $2.6 \times 10^{-5}$ Torr, corrected for xenon. The commercial gas feed system includes a 1000 -sccm mass flow controller for the anode and 200-sccm controller for the cathode with an uncertainty of measurement of $\pm 1.0 \%$ of reading. The commercial discharge power supply can provide up to $2000 \mathrm{~V}$ and $100 \mathrm{~A}$ with a $5.5 \mathrm{mF}$ output filter capacitance, and a $15.3 \mathrm{mF}$ capacitor bank was connected in parallel. Separate commercial power supplies were used to power the cathode heater, cathode keeper, and electromagnets. The thrust stand is an inverted pendulum thrust stand designed by Haag ${ }^{19}$ and is actively cooled during operation. The nominal accuracy is $\pm 2 \%{ }^{17}$ and thermal drift is corrected by measuring thrust signal periodically without gas flow, where the maximum was $\sim 10 \mathrm{mN}$. The thrust-to-power ratio and anode 
efficiency are defined $\operatorname{as}^{20}$

$$
\begin{gathered}
T / P=\frac{T}{P_{D}} \\
\eta_{a}=\frac{T^{2}}{2 \dot{m}_{a} P_{D}}
\end{gathered}
$$

where $T$ is thrust, $P_{D}=I_{D} V_{D}$ is the discharge power, and $\dot{m}_{a}$ is the anode mass flow rate. Anode efficiency is used in this investigation instead of total efficiency for ease of comparison with previous works.

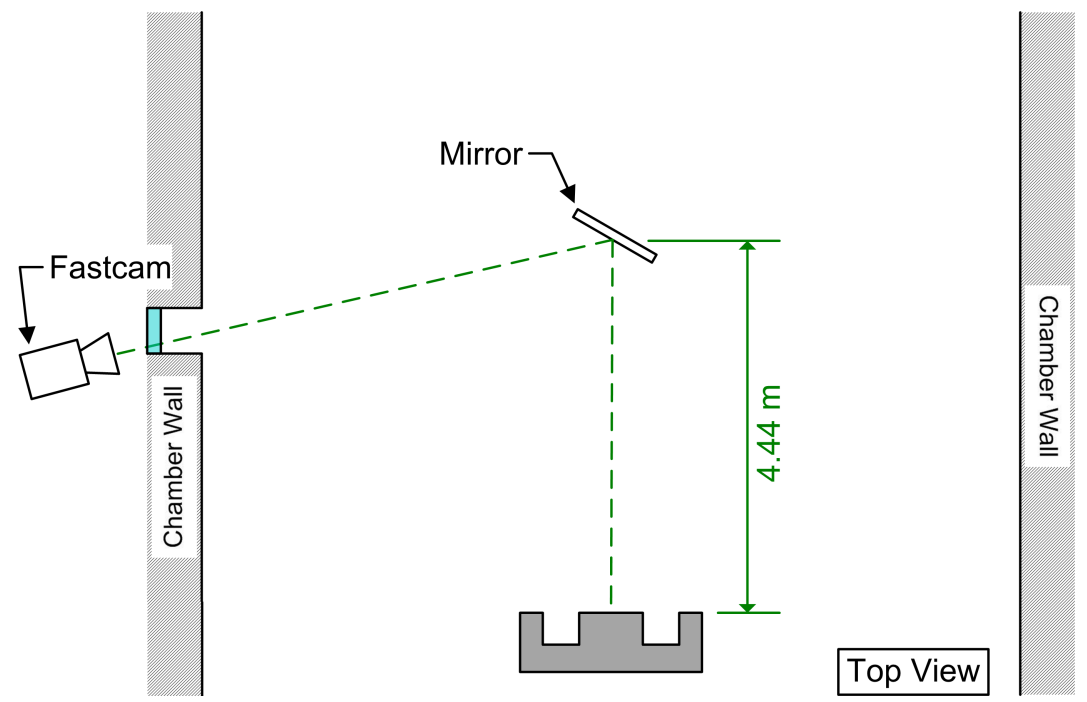

Figure 3. Experimental setup of 300MS-2 in VF-5 at NASA GRC showing thruster, mirror, and view port for high-speed imaging.

VF-5 does not have a view port on or close to thruster centerline. Therefore, the thruster was imaged from a side view port using a mirror $4.4 \mathrm{~m}$ downstream as shown in Figure 3. This caused the thruster image captured by the FastCam to be inverted, which was corrected in post-processing. Minor vibration was noticed in the mirror, but the frequency was lower than the plasma oscillations and did not perturb the data analysis. The same equipment and analysis techniques summarized in Section II.A and described in detail in Refs. 3 and 4 were used to acquire and process the HIA results presented here. An Agilent DSOX3024A digital oscilloscope with built-in function generator was used to trigger the FastCam and the oscilloscope to acquire discharge current data. The discharge current was measured with a Tektronix A6303 current probe connected between the capacitor bank and the thruster, and a Tektronix TM502A amplifier set to AC-coupled mode.

\section{B. Magnetically Shielded H6 (H6MS)}

\section{H6MS Information}

The H6MS shown in Figure 4 is based on the H6. The inner and outer front pole pieces and the inner and outer screens were modified to implement magnetic shielding. Changes were also made to the discharge channel with new erosion rings modified as described and shown in Figure 3 of Ref. 21. The design, detailed numerical modeling, and extensive testing of the H6MS have been well documented in a series of publications. ${ }^{5,6,21-23}$ During the initial test campaign to compare the H6MS to the H6, ${ }^{21}$ "practically erosion-free operation has been achieved for the first time in a high performance Hall thruster." It was noted that the specific impulse increased by $2.9 \%$, the total efficiency decreased by $1.7 \%$ and the insulator ring temperature decreased by 12-16\%. The discharge current oscillations increased by $25 \%$, but did not affect the thruster stability. The investigation presented in Ref. 21 used an anode mass flow rate of $18.35 \mathrm{mg} / \mathrm{s}$ with a cathode flow rate of $1.28 \mathrm{mg} / \mathrm{s}$. The present investigation uses an anode flow rate of $18.23 \mathrm{mg} / \mathrm{s}(<1 \%$ lower) with a cathode flow rate of $1.28 \mathrm{mg} / \mathrm{s}$. As shown in Figure 9(a), the discharge currents are the same to within $1 \%$ so this difference is negligible. 


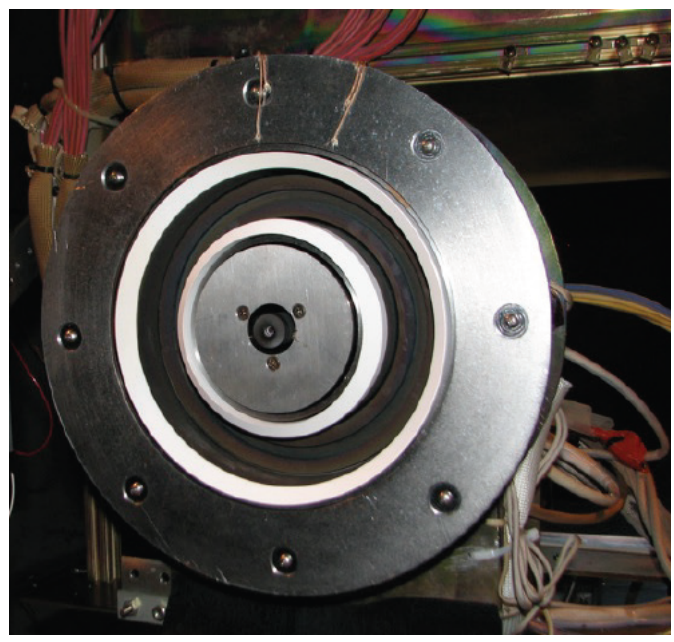

(a) On the thrust stand at NASA JPL. Reproduced from Figure 9 of Ref. 21.

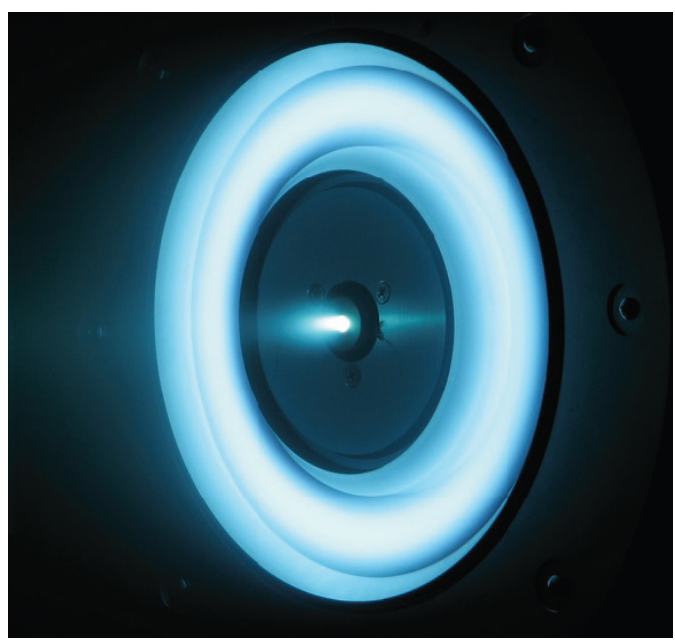

(b) Operating in the Owens Chamber at $6 \mathrm{~kW}$. Reproduced from Figure 7 of Ref. 21.

Figure 4. The H6MS at NASA JPL.

The same techniques of varying magnetic field strength without varying shape used in the $\mathrm{H} 6$ investigation $^{3,4}$ were employed here by maintaining a constant magnet coil current ratio of $I_{I M} / I_{O M}=1.21$. The magnetic field shape variations over magnet current settings were not verified with simulations like the H6 in the previous investigations. The magnetic field was known to become skewed due to saturation for $I_{I M} \geq 5 \mathrm{~A}^{\mathrm{a}}$, so a ratio of $I_{I M} / I_{O M}=1.00$ was investigated for the higher magnetic field settings. Although not shown here, the results are indistinguishable from the nominal coil current ratio, so $I_{I M} / I_{O M}=1.21$ is used for this investigation in order to provide a direct comparison with previous work. The implications of possible magnetic circuit saturation are discussed in Section V.A.

The first study on oscillations in the H6MS was conducted by Jorns ${ }^{24}$ using high-speed image analysis techniques similar to this investigation. Global oscillations in the entire discharge channel were observed in the frequency range of $7-12 \mathrm{kHz}$ that resemble the global oscillation mode for the unshielded $\mathrm{H} 6 .^{3,4}$ Additional oscillations were detected over the inner pole between the cathode and the discharge channel in the frequency range of $75-90 \mathrm{kHz}$ that were identified as cathode oscillations. Using linearized two-fluid equations in cylindrical coordinates, the cause for the cathode oscillations was determined to be density gradients near the centrally-mounted cathode. These waves propagate in the $E \times B$ direction in the $\mathrm{H} 6$ with a radial magnetic field pointing outwards, but they are gradient driven and not related to the azimuthal Hall current. The presence of these oscillations in the discharge current PSD of magnetically shielded thrusters suggests a strong coupling between the near cathode plasma and discharge plasma.

\section{Experimental Setup at NASA JPL}

This experiment was conducted in the Owens Chamber at NASA JPL, which is a $3 \mathrm{~m}$ diameter by $10 \mathrm{~m}$ long cryogenically pumped vacuum chamber. The pressure during testing was $1.2 \times 10^{-5}$ Torr corrected for xenon, as measured by an ion gauge mounted near the thrust stand. Similar to Figure 3 of Ref. 24, the FastCam is in a viewport of the Owens Chamber $\sim 7.5 \mathrm{~m}$ downstream and off thruster centerline by $\sim 5^{\circ}$. The FastCam acquisition and discharge current acquisition were triggered by a Wavetek 178 function generator sending a TTL pulse. A Tektronix DPO-3054 digital oscilloscope recorded the AC component of the discharge current at $500 \mathrm{kHz}$. Section IV of Ref. 21 contains additional details about the facility, the thruster and measurement diagnostics.

\footnotetext{
${ }^{\text {a}}$ Personal correspondence with R. Hofer, February 2014.
} 


\section{Results}

\section{A. 300MS-2 Results}

Magnetic field sweeps are completed for the 300MS-2 and two mode transitions are identified. During magnetic field sweeps, the magnet current increments are typically $0.5 \mathrm{~A}$, which is more coarse than the $0.1 \mathrm{~A}$ increments used for the $\mathrm{H} 6$ investigation near the transition points. ${ }^{3,4}$ As a result, transition regions could not be defined like the $\mathrm{H} 6$ investigation. A transition similar to the local-to-global mode transition in the unshielded $\mathrm{H} 6$ is observed at $I_{I M}=3.5 \mathrm{~A}$ where the discharge current and low frequency oscillations dominate for $I_{I M}<3.5 \mathrm{~A}$; however, no spokes are observed for $I_{I M}>3.5 \mathrm{~A}$. A new transition is observed between $I_{I M}=5.5-6.0$ A where there is no noticeable change in discharge current, but spokes are observed for $I_{I M} \geq 6.0 \mathrm{~A}$. For the discussion here, Mode 1 is $I_{I M}<3.5 \mathrm{~A}$, Mode 2 is $3.5 \leq I_{I M} \leq 5.75 \mathrm{~A}$ and Mode 3 is $I_{I M}>5.75 \mathrm{~A}$. The modes are summarized in Table 1 and their characteristics are detailed below.

\begin{tabular}{|c|c|c|c|}
\hline & Mode 1 & Mode 2 & Mode 3 \\
\hline Limits & $I_{I M}<3.5 \mathrm{~A}$ & $3.5<I_{I M}<5.75 \mathrm{~A}$ & $I_{I M}>5.75 \mathrm{~A}$ \\
\hline Description & $\begin{array}{l}\text { Global oscillation } \\
\text { (breathing mode) }\end{array}$ & Cathode oscillation & $\begin{array}{l}\text { Combined cathode, } \\
\text { spoke and breathing } \\
\text { mode }\end{array}$ \\
\hline $\begin{array}{l}\text { Low Frequency } \\
\qquad f<20 \mathrm{kHz}\end{array}$ & $\begin{array}{c}\text { Dominant } \\
1-5 \mathrm{kHz}(300 \mathrm{~V}) \\
5-10 \mathrm{kHz}(400 \mathrm{~V})\end{array}$ & $\begin{array}{l}\sim 5-10 \mathrm{kHz} \\
\text { oscillations present } \\
\text { but not dominant }\end{array}$ & $\begin{array}{c}\sim 5-10 \mathrm{kHz} \\
\text { oscillations present, } \\
\text { can be large than } \\
\text { cathode oscillations }\end{array}$ \\
\hline $\begin{array}{l}\text { High Frequency } \\
f=20-100 \mathrm{kHz}\end{array}$ & Weak or not present & $\begin{array}{c}\text { Dominant } \\
60 \mathrm{kHz}(300 \mathrm{~V}) \\
50-60 \mathrm{kHz}(400 \mathrm{~V})\end{array}$ & $\begin{array}{c}\text { Cathode related } \\
\text { oscillations at } 60 \mathrm{kHz}\end{array}$ \\
\hline Spokes & Not present & Not present & $\begin{array}{c}\text { Strong spokes; } \\
\mathrm{v}_{s p}=1000 \pm 100 \mathrm{~m} / \mathrm{s}\end{array}$ \\
\hline $\begin{array}{c}\text { Discharge Current } \\
\text { RMS/Mean }\end{array}$ & $>15 \%$ & $\begin{array}{c}<10 \%(300 \mathrm{~V}) \\
10-30 \%(400 \mathrm{~V})\end{array}$ & $\begin{array}{c}\sim 10 \%(300 \mathrm{~V}) \\
10-20 \%(400 \mathrm{~V})\end{array}$ \\
\hline$I_{D}$ & High & Low & Middle \\
\hline$T$ & Low & Middle & High \\
\hline$T / P$ & Low & High & Middle \\
\hline$\eta_{a}$ & Low & High & Middle \\
\hline
\end{tabular}

Table 1. Summary table of modes and oscillations for the 300MS-2. The bottom four rows show a qualitative ranking for $I_{D}, T, T / P$ and $\eta_{a}$ with green, yellow, and red showing progression from more to less favorable.

Figure 5 shows the discharge current, thrust, thrust-to-power, and anode efficiency for the 300 and $400 \mathrm{~V}$ conditions. Figure 6 shows the discharge current oscillation amplitude defined as the RMS value divided by the mean value, $\widetilde{I}_{D} / \bar{I}_{D}$. Except for thrust, the same trends as the unshielded $\mathrm{H} 6$ are observed for the 300MS-2. ${ }^{3,4}$ The 300MS-2 shows a general decrease in thrust with decreasing magnetic field magnitude in Figure 5(b), which is different than the $\mathrm{H} 6$ where thrust was nearly constant. The transition from Mode 2 to Mode 1 is very similar to the local to global mode transition observed in the unshielded H6 with a sharp increase in mean discharge current (Figure 5(a)) and oscillation amplitude (Figure 6). Mode 1 shows the highest discharge current, lowest thrust, lowest thrust-to-power and lowest anode efficiency. The performance values are maximum in Mode 2 where the discharge current is minimum, the thrust-to-power peaks, and the anode efficiency peaks. The $400 \mathrm{~V}$ condition shows high-amplitude discharge current oscillations in Figure 5(a) and Figure 6 near the spoke transition point that is discussed in Section V.C. The thrust peaks in Mode 3, but the discharge current is higher than in Mode 2 so the thrust-to-power is less than in Mode 2. In general, thrust in Figure 5(b) peaks near the spoke mode transition, but the mean discharge current in Figure 5(a) and discharge current oscillation amplitude in Figure 6 are unaffected by the spoke mode transition. The observation that the presence or absence of spokes does not affect the discharge current 
between Modes 2 and 3 could be an important clue in understanding the nature of spokes in magnetically shielded thrusters.

(a) Discharge Current

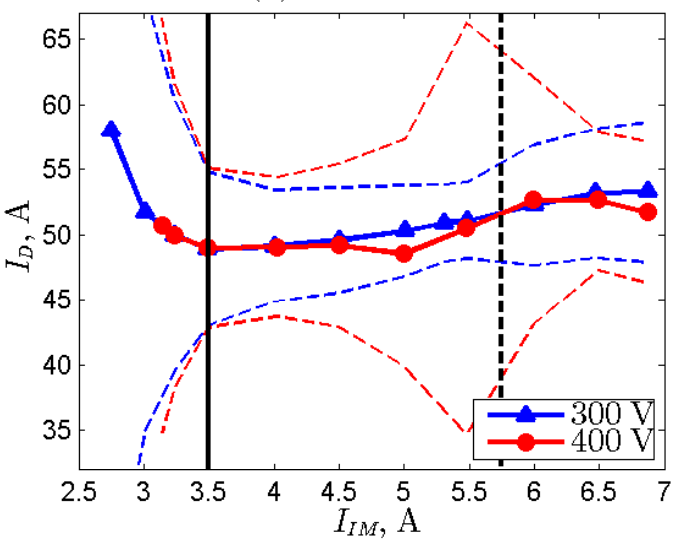

(c) Thrust to Power

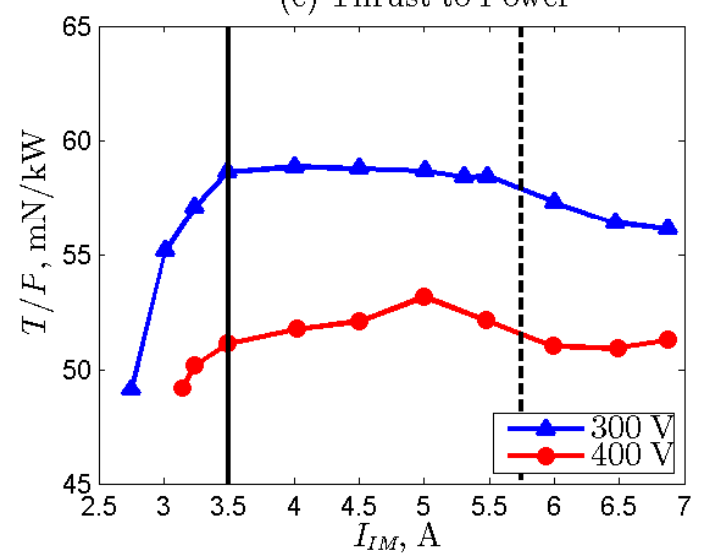

(b) Thrust

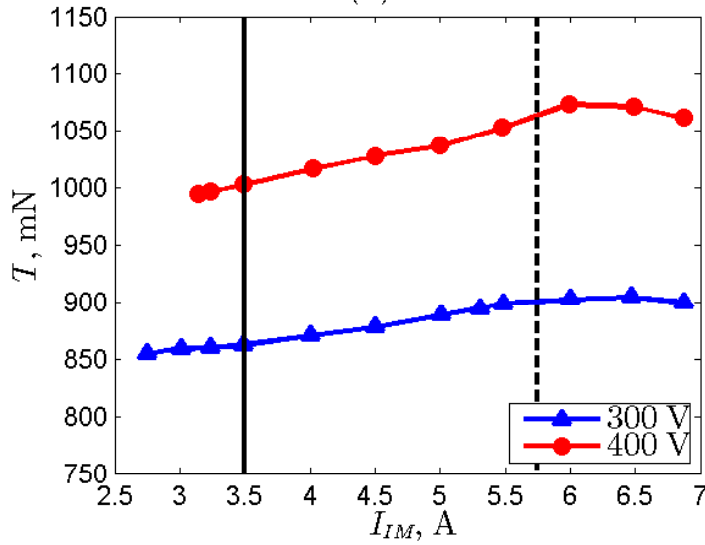

(d) Anode Efficiency

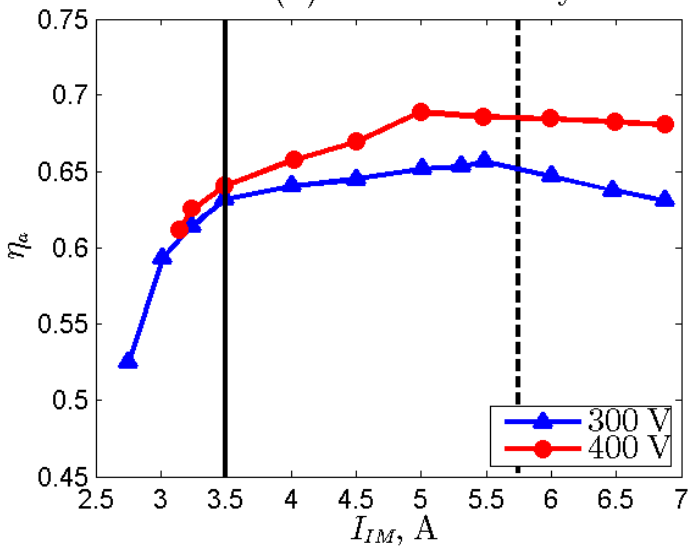

Figure 5. 300MS (a) discharge current, (b) thrust, (c) thrust-to-power, and (d) anode efficiency for the $300 \mathrm{~V}$ (blue) and $400 \mathrm{~V}$ (red) conditions during B-field sweeps. The dashed lines around $I_{D}$ are the discharge current oscillation amplitude or RMS values. The two mode transition lines are also shown.

B-field sweeps and HIA for the $300 \mathrm{~V}$ and $400 \mathrm{~V}$ conditions are shown in Figures 7 and 8, respectively. The top plot shows the mean discharge current with the RMS values as dashed lines around the mean to visualize oscillation amplitude. The selected magnet settings shown are $I_{I M}=3.0$ or $3.1,4.5$ and $6.5 \mathrm{~A}$, which correspond to oscillation Mode 1, 2 and 3, respectively, as described in Table 1. The second row plots are the HIA PSDs and the discharge current PSDs plotted on different axes. The $I_{D}$ PSD corresponds to the left axis while $m_{0}$ and spoke orders $m=2-9$ correspond to the right axis. The different scaling is due to different units of the original signals (current in A versus current density in $\mathrm{mA} / \mathrm{cm}^{2}$ ). The traces overlay each other when scaled as shown in Figures 7 and 8, which reinforces the correlation between $I_{D}$ and $m_{0}$ that has been shown previously. The HIA upper frequency limit is $43.75 \mathrm{kHz}$ due to the camera frame rate of $87.5 \mathrm{kHz}$, but the discharge current sampling rate allowed the upper limit on $I_{D}$ PSD to be over $80 \mathrm{kHz}$. This is important because the cathode oscillations between $50-60 \mathrm{kHz}$ cannot be resolved by HIA (with the Photron SA5 at $256 \times 256$ resolution), but they can be seen in the discharge current. The third row plots in Figures 7 and 8 are discharge current density, $j$, calculated using the HIA techniques summarized in Section II.A. The fourth row is a normalized spoke surface that is calculated by normalizing each frame (vertical column of points or $\theta=0 \rightarrow 360^{\circ}$ at a given time, $t_{i}$ ) from the discharge current density according to

$$
\hat{j}_{i}\left(\theta, t_{i}\right)=\frac{j\left(\theta, t_{i}\right)-\bar{j}\left(t_{i}\right)}{\widetilde{j}\left(t_{i}\right)} .
$$

where $\bar{j}$ is the mean value and $\widetilde{j}$ is the RMS value. This technique isolates and intensifies any azimuthal 


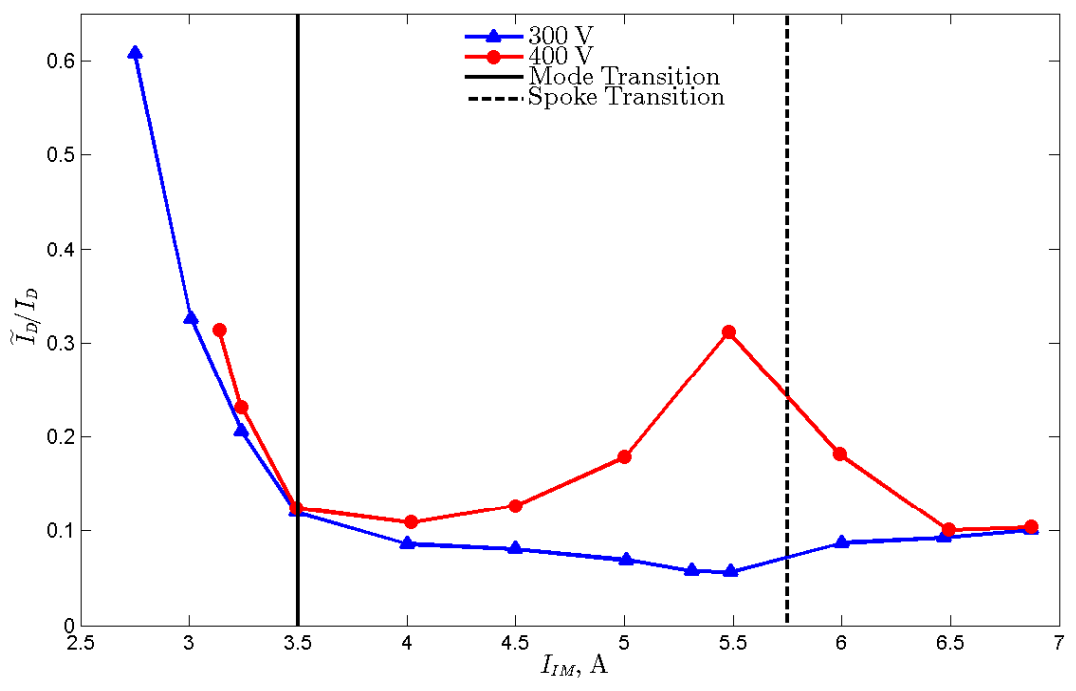

Figure 6. Discharge current oscillation amplitude for 300MS-2 during magnetic field sweeps for $300 \mathrm{~V}$ (blue) and $400 \mathrm{~V}$ (red). Oscillation amplitude is the RMS value divided by the mean value. Mode transition lines are shown.

spokes that may be present by eliminating entire channel oscillations. When spokes are not present, the normalized spoke surfaces appear as nearly random noise.

In Figure 7, the lowest magnetic field setting of $I_{I M}=3.0 \mathrm{~A}$ corresponds to Mode 1 in Table 1 and shows the breathing mode similar to the unshielded thruster. There is a strong peak at $3 \mathrm{kHz}$, a lower peak at $24 \mathrm{kHz}$ and another broad peak from 56-60 kHz. No spokes are present in the PSD. The discharge current density shows large amplitude global oscillations from 50 to $250 \mathrm{~mA} / \mathrm{cm}^{2}$. The indeterminate, noisy nature of the normalized spoke surface reinforces that no spokes are present. The discharge current oscillation amplitude is the largest in this mode and is $>20 \%$ of the mean as illustrated in Figure 6, and increases with decreasing magnetic field strength starting at the mode transition line.

The magnetic field setting of $I_{I M}=4.5 \mathrm{~A}$ in Figure 7 corresponds to Mode 2 in Table 1 and shows that cathode oscillations are dominant. The peak at $\sim 60 \mathrm{kHz}$ is larger than the breathing mode peak at $\sim 5 \mathrm{kHz}$. Again, no spokes are present in the PSD, which is reinforced by the noisy normalized spoke surface. Oscillations in $j$ are smaller than in Mode 1 and vary between 115 and $140 \mathrm{~mA} / \mathrm{cm}^{2}$; however, the cathode oscillations are higher frequency than the Nyquist limit of the FastCam so the oscillations are not fully resolved. The discharge current oscillation amplitude is the least of all modes at less than $10 \%$ in Mode 2 for $300 \mathrm{~V}$, as shown in Figure 6, with the minimum at $I_{I M}=5.5$ A close to the spoke transition line. It is interesting to note that unshielded thrusters also show cathode oscillations in the plume region over the inner pole, but their influence is not typically detected in the discharge current signal. ${ }^{4}$ Therefore, it is possible that magnetically shielded thrusters are more susceptible to oscillations from plasma conditions outside the discharge channel.

The highest magnetic field setting of $I_{I M}=6.5 \mathrm{~A}$ in Figure 7 corresponds to Mode 3 in Table 1 and simultaneously shows the breathing mode, spokes, and cathode oscillations. The $I_{D}$ PSD shows a peak at $\sim 3 \mathrm{kHz}$ that corresponds to the breathing mode and a broad peak at $\sim 60 \mathrm{kHz}$ that corresponds to cathode oscillations. Spokes are clearly present by the strong peaks in $m=2-9$ of the HIA PSD. The strongest peak is $m=3$, which is over an order of magnitude higher than all other spokes orders. The normalized spoke surface shows that only 3 spokes are present at one time. Angular striations can be seen in $j$ intermixed with vertical lines showing a mixture of whole-channel oscillations and azimuthal spokes that are between 110 and $160 \mathrm{~mA} / \mathrm{cm}^{2}$. The normalized spoke surface isolates the spokes very clearly with a spoke velocity of $\mathrm{v}_{s p}=1000 \pm 100 \mathrm{~m} / \mathrm{s}$ calculated using the techniques described in Ref. 12. The discharge current oscillation amplitude shown in Figure 6 is $\sim 10 \%$ in Mode 3 for $300 \mathrm{~V}$.

Figure 8 shows many of the same trends for the $400 \mathrm{~V}$ condition as the $300 \mathrm{~V}$ condition. In Mode 1 a strong breathing mode peak is observed at $5.5 \mathrm{kHz}$ that is evidenced by strong vertical lines in $j$ with oscillations between 50 and $250 \mathrm{~mA} / \mathrm{cm}^{2}$. The normalized spoke surface and the HIA do not show any 


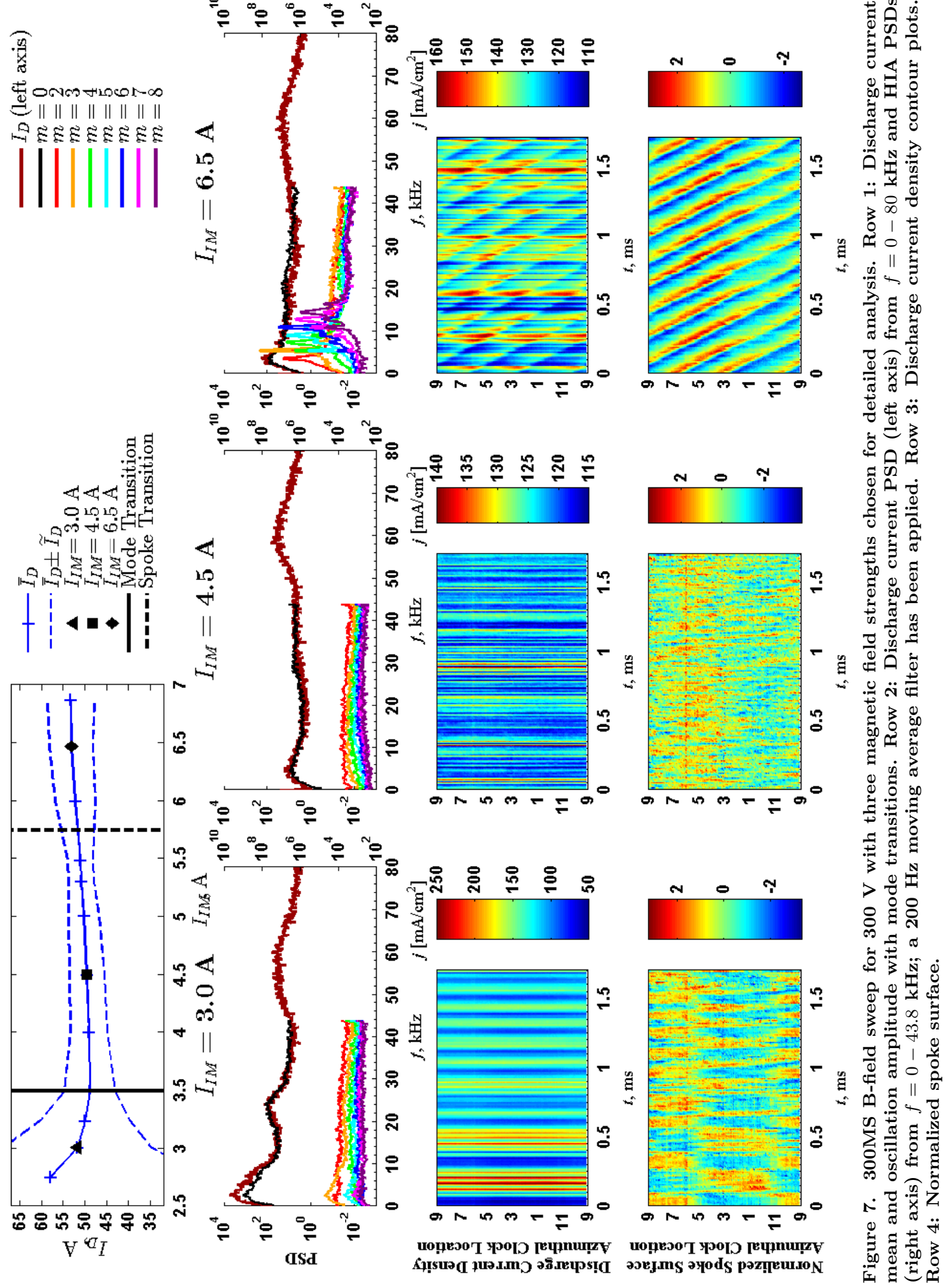




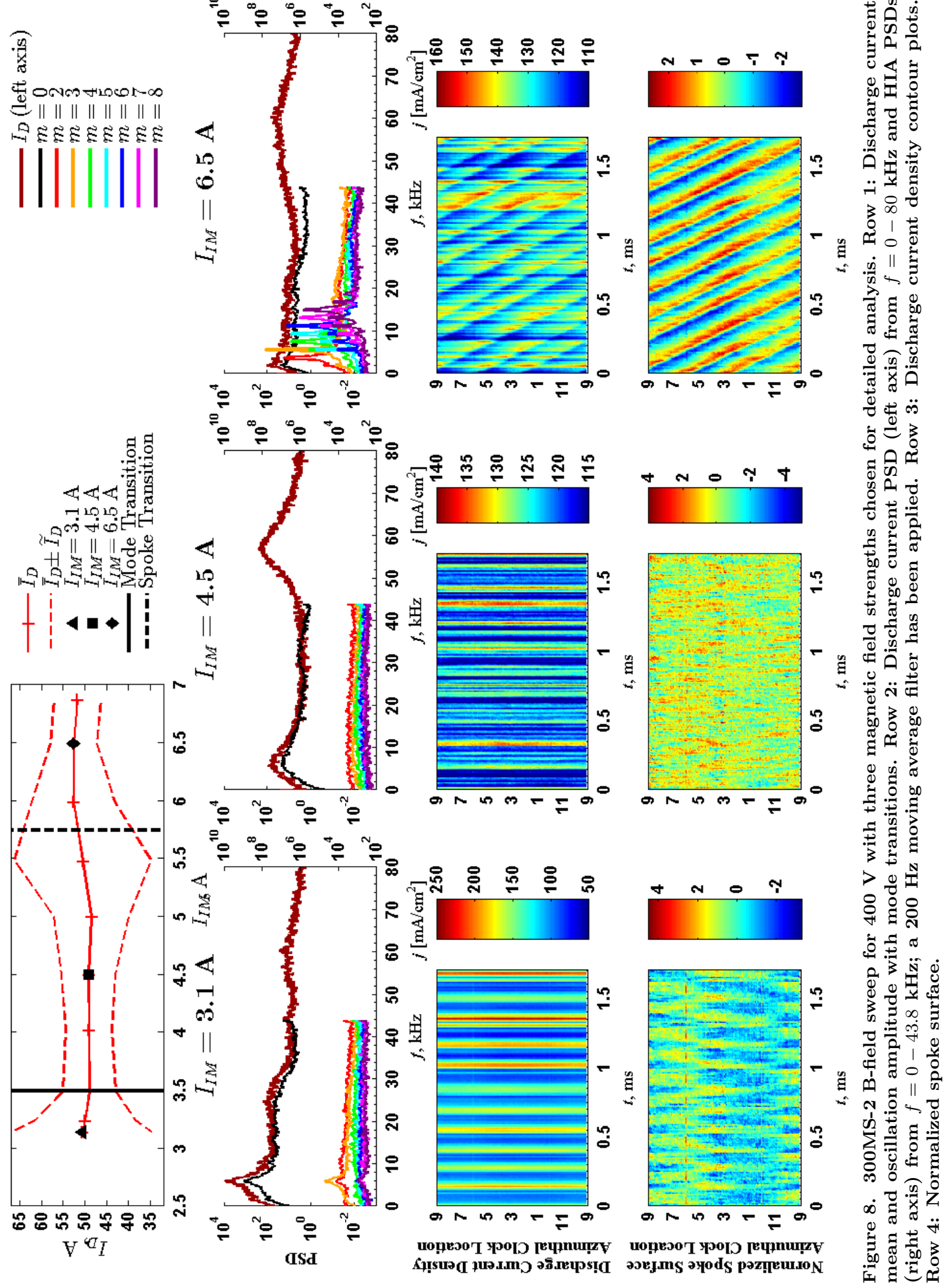


evidence of spokes. In Mode 2 the cathode oscillations dominate at $57 \mathrm{kHz}$ with breathing mode oscillations at $6 \mathrm{kHz}$ of lower magnitude. The discharge current density oscillation amplitudes are smaller between 115 and $140 \mathrm{~mA} / \mathrm{cm}^{2}$. As with Mode 1, the normalized spoke surface and the HIA do not show any evidence of spokes. In Mode 3 a combination of breathing mode, cathode oscillations and spokes are present. Spokes are clearly visible in the HIA PSD with the dominant spoke order of $m=3$ and three spokes observed in the normalized spoke surface. The breathing mode peak is $\sim 2 \mathrm{kHz}$ and is the same strength as the broad cathode oscillations from $50-60 \mathrm{kHz}$.

\section{B. H6MS Results}

The discharge current with oscillation amplitude (RMS) is shown in Figure 9(a) for a $300 \mathrm{~V}$ discharge. Only discharge current was measured in the present work, but matched well with data from the initial investigation by Hofer ${ }^{21}$ so the thrust data in Figure 10 of that work is applicable here and reproduced in Figure 9(b). Similar to the 300MS-2, three modes are observed with two transition points at approximately $I_{I M} \approx 2.75 \mathrm{~A}$ and $I_{I M} \approx 5.25 \mathrm{~A}$. The performance data for the H6MS in Figure 9 shows the same trends as the 300MS-2 in Figure 5.

(a) Discharge Current

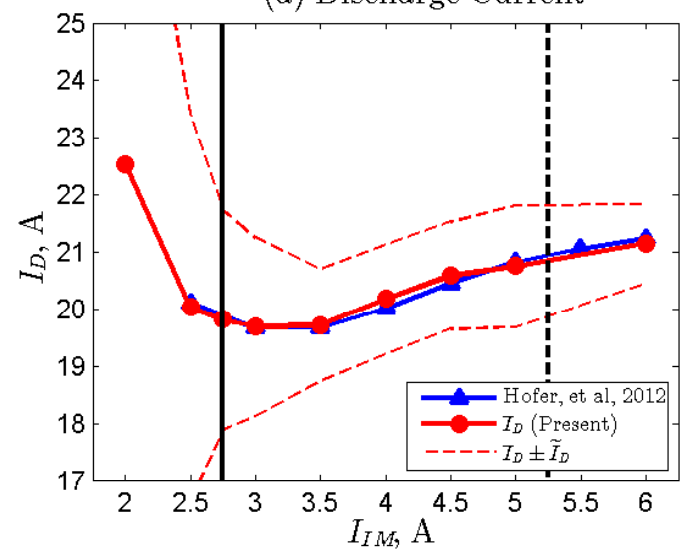

(c) Thrust to Power

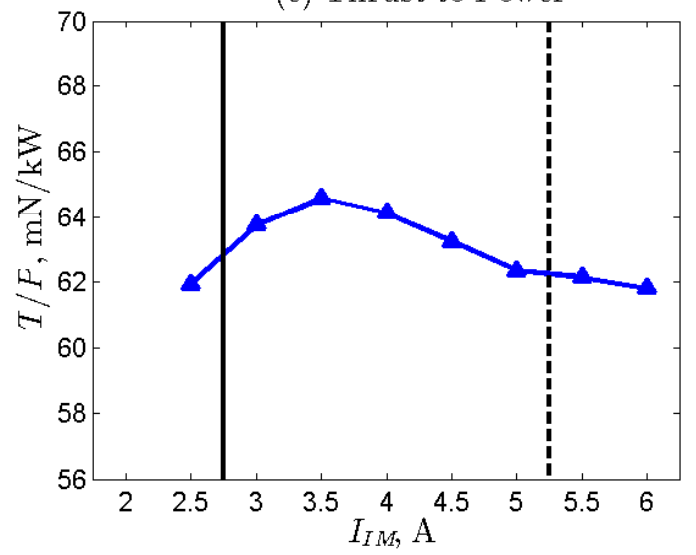

(b) Thrust

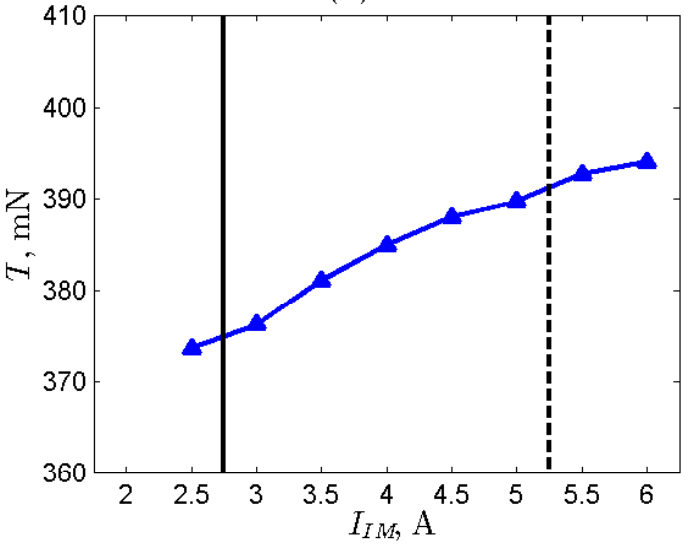

(d) Anode Efficiency

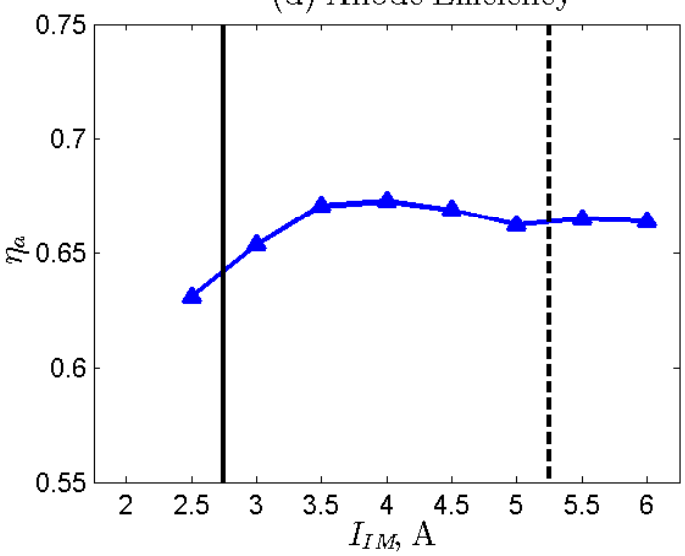

Figure 9. H6MS (a) discharge current, (b) thrust, (c) thrust-to-power, and (d) anode efficiency for the $300 \mathrm{~V}$ condition (blue) reproduced from Figure 10 of Ref. 21 and the present work (red) during B-field sweeps. Only discharge current was measured in the present work, but matched well with the previous investigation so the performance data is valid. The dashed lines around $I_{D}$ are the discharge current oscillation amplitude or RMS values. The two mode transition lines are also shown. The nominal magnet coil current is $I_{I M}=4.0 A$.

The discharge current in Figure 9(a) is minimized in Mode 2 and shows a minimum value at $I_{I M}=3.5 \mathrm{~A}$, which is a lower setting than the nominal setting of $I_{I M}=4.0 \mathrm{~A}$. The thrust in Figure 9(b) continually decreases with decresing B-field and a $5 \%$ decrease from the peak in Mode 3. The thrust-to-power in Figure 9(c) shows a peak in Mode 2 at a lower B-field setting than the nominal setting. The total efficiency 
is presented in Figure 10 of Ref. 21, but the anode efficiency shown in Figure 9(d) was calculated from the discharge current in Figure 9(a), thrust in Figure 9(b), an anode mass flow rate of $18.35 \mathrm{mg} / \mathrm{s}$, and Equation 2. Similar to the 300MS-2, the anode efficiency peaks in Mode 2.

Figure 10 shows the H6MS magnetic field sweep at the $300 \mathrm{~V}$ condition with HIA. Similar to the 300MS-2 data in Figure 7, three modes are observed with two transition points. Select magnetic field strengths have been chosen for further analysis in each of the modes. Figure 10 can be compared to the unshielded H6 $300 \mathrm{~V}$ sweep in Figure 1. In Mode 1 at $I_{I M}=2.5 \mathrm{~A}$, the breathing mode at $10 \mathrm{kHz}$ is an order of magnitude higher than the cathode oscillations at $69 \mathrm{kHz}$ with no detectable spokes in the normalized spoke surface. Although not shown, the cathode oscillations nearly disappear at $I_{I M}=2.0 \mathrm{~A}$, which is deep into breathing mode. The discharge current density oscillates globally around the discharge channel with fluctuations between 100 and $150 \mathrm{~mA} / \mathrm{cm}^{2}$ with some spikes up to $180 \mathrm{~mA} / \mathrm{cm}^{2}$.

The nominal condition at $I_{I M}=4.0 \mathrm{~A}$ is in Mode 2 and also does not exhibit any detectable spokes in the normalized spoke surface. The breathing mode frequency remains at $10 \mathrm{kHz}$ and is approximately the same strength as the cathode oscillations. The cathode oscillations peak at $78 \mathrm{kHz}$, but the peak width is broader than in Mode 1 before they disappear. The discharge current density oscillates globally around the discharge channel with fluctuations between 120 and $140 \mathrm{~mA} / \mathrm{cm}^{2}$ with some spikes up to $150 \mathrm{~mA} / \mathrm{cm}^{2}$. However, the cathode oscillations are above the Nyquist frequency of the FastCam so these oscillations are not adequately captured in the HIA PSD or spoke surfaces.

Mode 3 at $I_{I M}=6.0$ A shows a strong $m=3$ spoke order in the HIA PSD, which can be observed as three bright regions on any vertical line of the normalized spoke surface. The $m=3$ peak is over an order of magnitude higher than the next highest $m=6$, which is a harmonic, and 2 orders of magnitude higher than other spoke orders. Using the correlation method described in Ref. 12, the spoke velocity is $1450 \pm 170 \mathrm{~m} / \mathrm{s}$. The cathode oscillation peak is even broader in Mode 3 and the approximate peak is at $68 \mathrm{kHz}$. The discharge current PSD has a broader peak between 7 and $10 \mathrm{kHz}$ and is the same strength as the cathode oscillations. The discharge current density displays spokes (diagonal striations) and global oscillations (vertical lines) with the fluctuations between 130 and $150 \mathrm{~mA} / \mathrm{cm}^{2}$.

Figure 11 shows the same B-field sweep as Figure 10, but the selected magnetic field settings for further analysis are within Mode 2, $I_{I M}=3.0,4.0$ and 5.0 A. These represent the limits before the upper and lower transition as well as the nominal setting of $I_{I M}=4.0 \mathrm{~A}$. The discharge current increases with increasing magnetic field strength, $\bar{I}_{D}=19.7,20.2$ and $20.8 \mathrm{~A}$, respectively. The breathing mode oscillations remain the same strength, but the frequency decreases with increasing magnetic field strength, $f=10.3,9.1$, and $6.8 \mathrm{kHz}$, respectively. The cathode oscillations show a significant change in response to the magnetic field strength. The cathode oscillation strength decreases from nearly the same strength as the breathing mode near the lower transition point to barely detectable near the upper transition point. The frequency peaks are 75,77 and a barely perceptible peak near $70 \mathrm{kHz}$, respectively. No spokes are observed in the normalized spoke surface.

\section{Discussion}

\section{A. Magnetic Field Simulations}

Conducting magnetic field simulations across the range of coil currents tested is important to ensure the topology is characterized for each setting and does not deviate significantly from the nominal shape. Section III stated that magnetic field simulations were not performed across the range of magnet settings for the $300 \mathrm{MS}-2$ or the H6MS as was done for the unshielded H6 investigation. If the magnetic circuit is saturated at high coil currents, then the magnetic field could be skewed causing deviations from the topology shown schematically in Figure 1(c) of Ref. 5. These deviations could cause field lines to intersect the discharge channel walls thus increasing plasma contact with the walls, which is minimized in nominal magnetically shielded configurations. Therefore, unintentional plasma contact with the channel walls at high coil currents could be the cause for azimuthal spokes observed in Mode 3. This potentially indicates plasma-wall interactions play a role in the formation and propagation of azimuthal spokes. This finding would be significant in that spokes could be turned on or off through magnetic field variations. It may provide researchers the ability to study the role of plasma-wall interactions in HETs by intentionally forcing the plasma to contact the wall (unshielded) or have reduced plasma-wall contact (shielded) by changing the B-field during operation. This is the subject of on-going research and will be presented in future publications. 


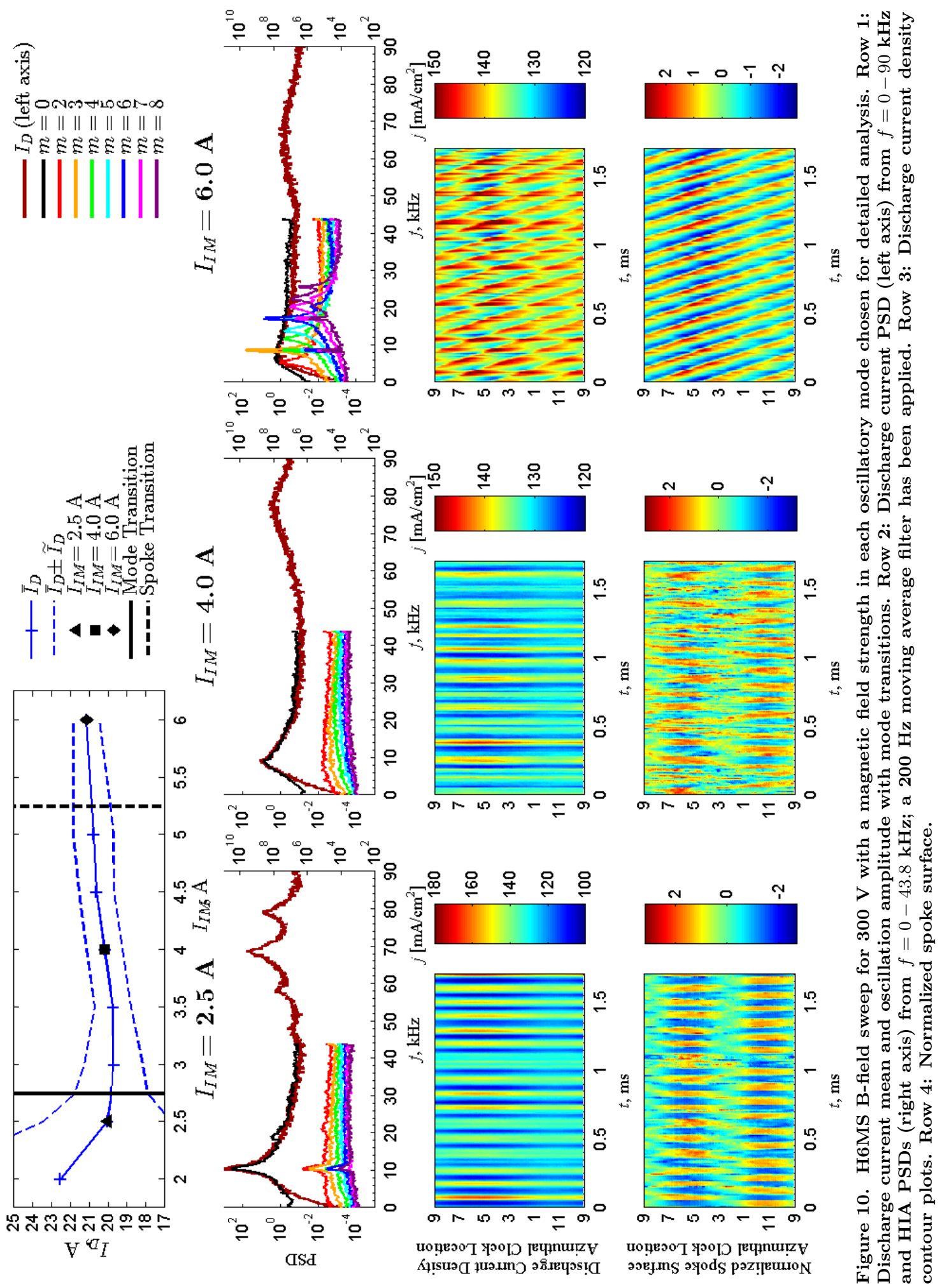



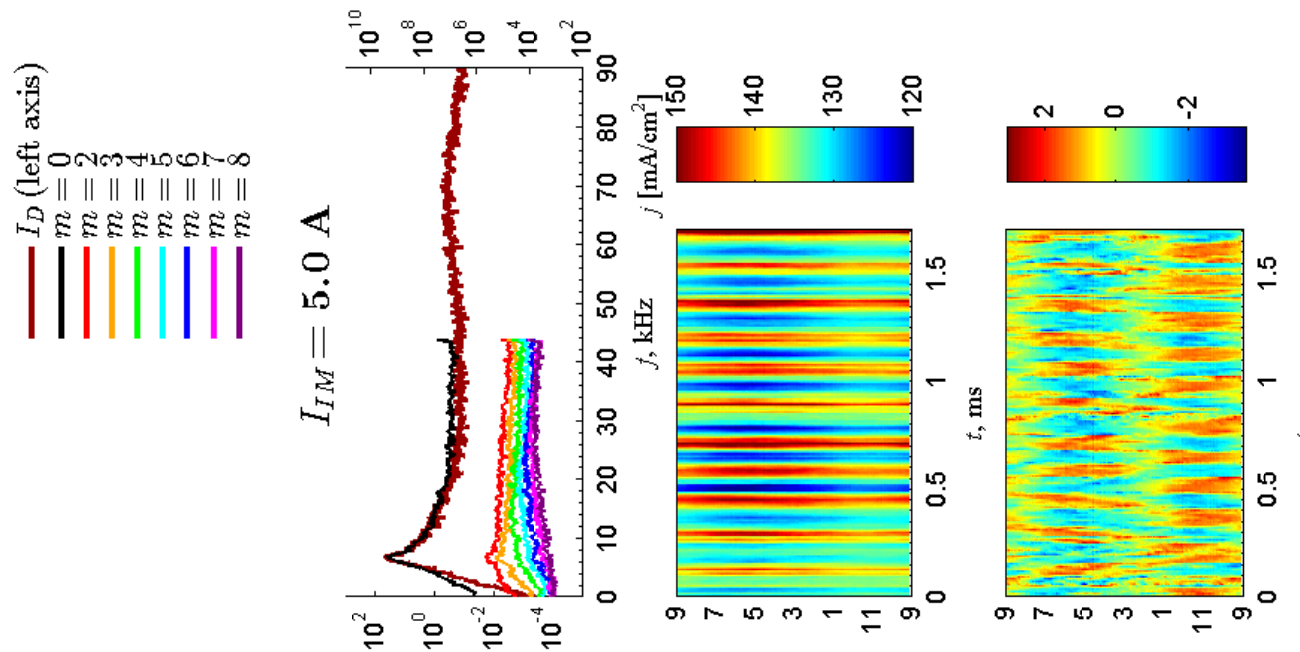

สี

듕

$\ddot{1}$

$\ddot{7}$

8 \&

니잉

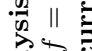

ส ह

न

สิ $\frac{0}{x}$

क

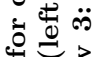

०

की

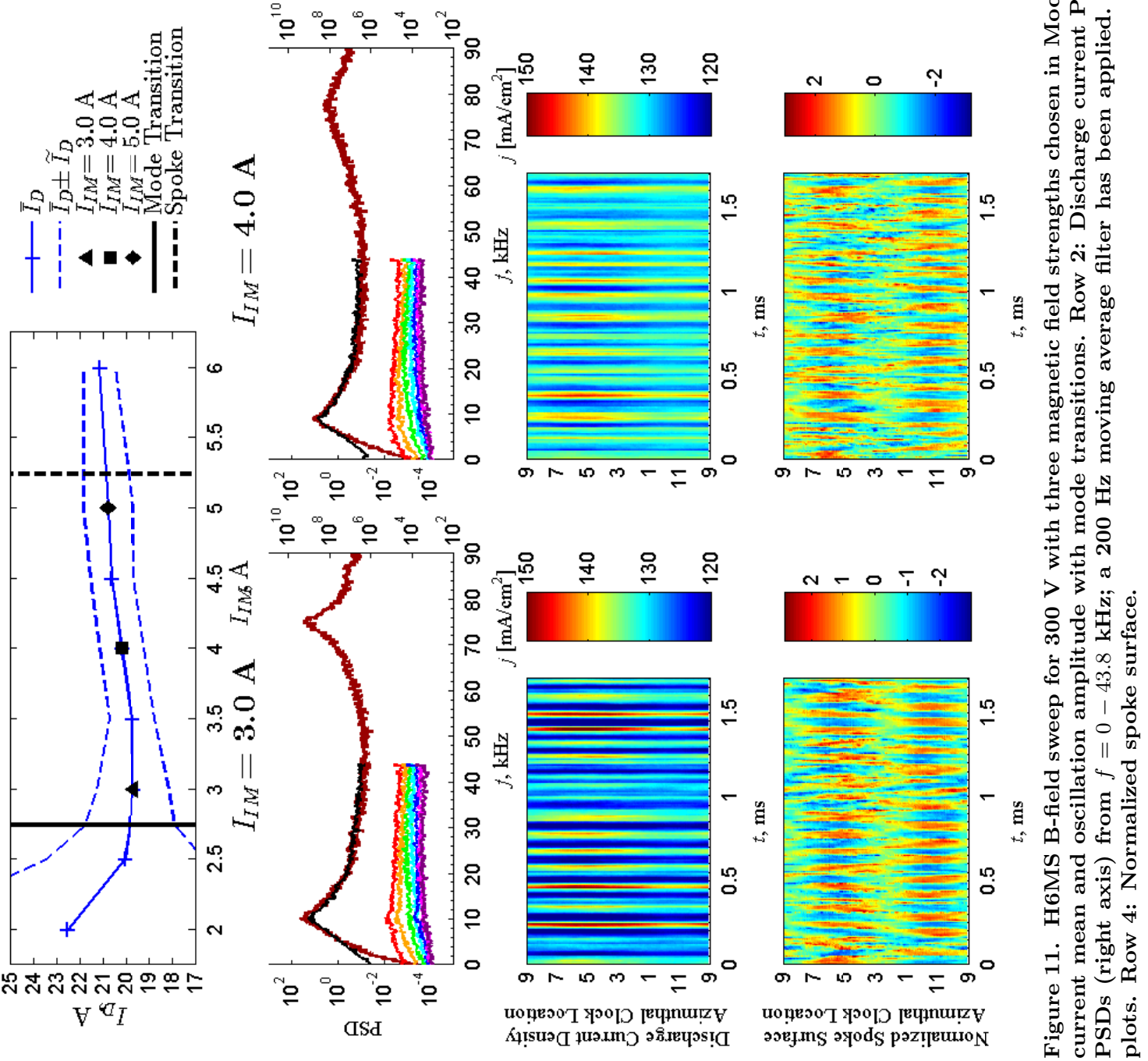




\section{B. Spokes and Cross Field Transport}

Both magnetically shielded thrusters exhibit two transition points with three different oscillatory modes, and the discharge current responds differently at each transition point. When spokes appear at the higher transition point, the mean discharge current is unaffected. At the lower transition point, the discharge current increases significantly similar to the local-to-global mode transition of unshielded thrusters. Previous work by Brown ${ }^{11}$ showed that the local-to-global mode transition corresponded to an increase in electron transport through the channel for unshielded thrusters, and it is reasonable to assume similar mechanisms are responsible for the increased discharge current in magnetically shielded thrusters at the lower transition point. The discharge current remained unchanged between Mode 2 and 3 at the upper transition point suggests that the appearance of spokes does not measurably affect cross field transport in magnetically shielded thrusters.

\section{300MS-2 Cathode Oscillation Resonance}

The most significant difference between the $300 \mathrm{~V}$ and $400 \mathrm{~V}$ conditions is the very sharp peak in discharge current oscillation amplitude for $400 \mathrm{~V}$ at $I_{I M}=5.5 \mathrm{~A}$, which corresponds to a minimum in oscillation amplitude for $300 \mathrm{~V}$ as shown in Figure 6. This trend of large discharge current oscillation amplitude crossing the spoke mode transition boundary from Mode 2 to Mode 3 suggests the spokes are unrelated to this phenomenon. Surprisingly, Figure 5(a) shows that mean discharge current is unaffected by these increased oscillations. This indicates it is not a global or breathing mode oscillation since those are associated with increased mean discharge current as well as increased oscillation amplitude. The PSDs for different magnet settings for $300 \mathrm{~V}$ and $400 \mathrm{~V}$ are shown in Figure 12. The $300 \mathrm{~V}$ condition in Figure 12(a) shows the $3-5 \mathrm{kHz}$ breathing mode oscillation is present for Modes $1-3$. There is always a $50-60 \mathrm{kHz}$ peak for cathode oscillations that is only higher in magnitude than the breathing mode for Mode 2 . The $400 \mathrm{~V}$ condition in Figure 12(b) shows the $2-6 \mathrm{kHz}$ breathing mode oscillation is present for Modes 1-3. There is also always a $50-60 \mathrm{kHz}$ peak for cathode oscillations that is higher in magnitude than the breathing mode for Mode 2. The high amplitude oscillations observed for the $400 \mathrm{~V}$ condition in Mode 2 at $I_{I M}=5.5 \mathrm{~A}$ are cathode oscillations strongly peaked at $59 \mathrm{kHz}$. The oscillation amplitude (Figure 6 ) and oscillation strength (Figure 12(b)) are nearly the same as the breathing mode in Mode 1. However, these oscillations do not affect thruster performance as shown in Figure 5. The 300MS appears to be more susceptible to cathode oscillations at $400 \mathrm{~V}$ that do not impact performance. The magnetic field setting of $I_{I M}=5.5 \mathrm{~A}$ at $400 \mathrm{~V}$ appears to be a resonance with cathode oscillations and merits further investigation.
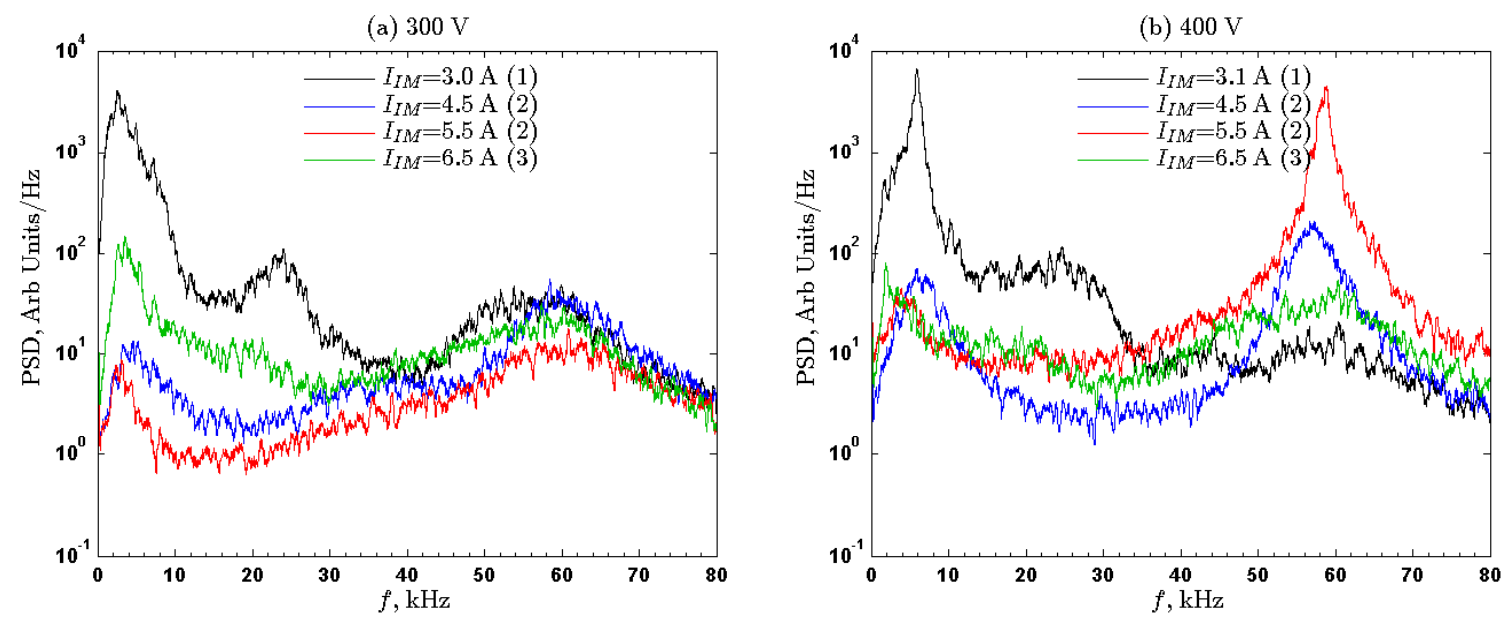

Figure 12. Discharge current PSD for the 300MS-2 at (a) $300 \mathrm{~V}$ and (b) $400 \mathrm{~V}$ for selected magnet settings. Mode numbers are shown in parentheses. All PSDs have been smoothed by a $400 \mathrm{~Hz}$ moving average filter. 


\section{Comparison between $\mathrm{H} 6$ and H6MS}

The mean discharge current and oscillation amplitude for the H6 and H6MS at $300 \mathrm{~V}$ are compared in Figure 13. The flow rate for the H6 and H6MS are $19.5 \mathrm{mg} / \mathrm{s}$ and $18.23 \mathrm{mg} / \mathrm{s}$, respectively. The upper and lower bounds of the transition region between global and local mode are shown for the H6. The transition points between Modes 1, 2 and 3 are shown for the H6MS. The magnetic field strength has been normalized by the nominal values of inner magnet coil current of $I_{I M}=3.50 \mathrm{~A}$ for the $\mathrm{H} 6$ and $I_{I M}=4.00 \mathrm{~A}$ for the H6MS as used in Ref. 21. The discharge currents show remarkable similarity with the largest difference at the highest magnetic field settings. The H6MS mean discharge current is larger at the higher magnetic field settings while the $\mathrm{H} 6$ oscillation amplitude is larger. The transition from global-to-local mode for the H6MS occurs at higher relative magnetic field strength than when the $\mathrm{H} 6$ enters the transition region to global mode, however the uncertainty on the H6MS transition point is $\pm 0.05 B_{r} / B_{r}^{*}$ due to larger $I_{I M}$ increments. The mean discharge current values do not differ significantly for $B_{r} / B_{r}^{*}<1$ regardless of the slight difference in transition point. The $\mathrm{H} 6$ shows less change in the discharge current above the transition point $\left(\Delta I_{D} \sim 0.5 \mathrm{~A}\right)$ than the H6MS $\left(\Delta I_{D} \sim 1.5 \mathrm{~A}\right)$. Ref. 21 showed that magnetic shielding had minimal impact on thruster operation, which is reinforced by this investigation and similarities in mode transitions between the H6 and H6MS for global-to-local mode and Mode 1 to Mode 2, respectively.

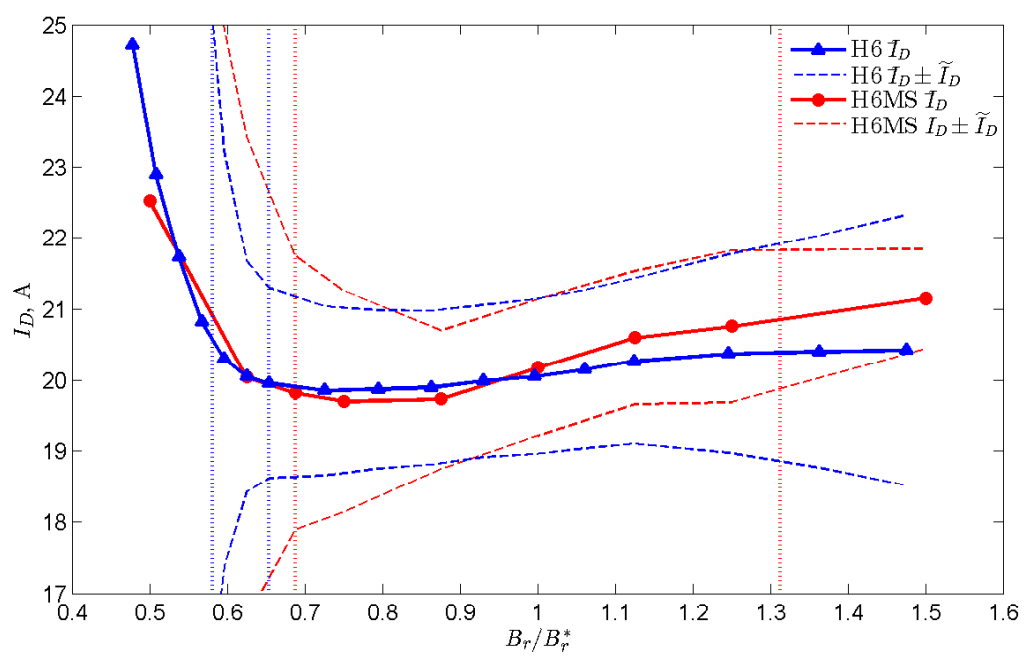

Figure 13. Comparison of discharge current for the H6 (blue) and H6MS (red) during a B-field sweep for $300 \mathrm{~V}$ operation. The oscillation amplitude (RMS) is shown above and below the mean value. Vertical blue lines are the boundaries for the transition region between local mode and global mode for the H6 identified in Ref. 4. Vertical red lines are the transition points for the H6MS between Mode 1, 2 and 3.

Selected discharge current PSDs from the H6 mode transition investigation of Ref. 4 are shown in Figure. 14. Three magnetic field settings have been selected from three different B-field sweeps at the $300 \mathrm{~V}$, $19.5 \mathrm{mg} / \mathrm{s}$ condition to identify test variability. Sweeps 1 and 2 were conducted by decreasing $B r / B_{r}^{*}$, while Sweep 3 was conducted by increasing $B_{r} / B_{r}^{*}$. Sweeps 2 and 3 were conducted consecutively during the same pump-down. Referencing the selected magnetic field settings in Figure 1 of Section II, the magnetic field settings shown in Figure. 14 are $B_{r} / B_{r}^{*}=0.52,1.00$ and 1.48 corresponding to global mode, local mode at the reference magnetic field strength, and local mode at high magnetic field strength, respectively. Note that the PSDs in Figure 10 for the H6MS have different scale factors than in Figure 14 for the H6, therefore it is not immediately obvious which thruster exhibits stronger oscillations. However, PSDs in Figure 12 from Ref. 21 show the H6MS has stronger oscillations than the H6.

Global mode $\left(B_{r} / B_{r}^{*}=0.52\right)$ in Figure. 14 exhibits strong oscillations sharply peaked at $9 \mathrm{kHz}$ with higher harmonics also observed for Sweeps 2 and 3. In addition, there are $50-70 \mathrm{kHz}$ oscillations for all sweeps that are three orders of magnitude lower and the correct frequency range to be cathode oscillations. This PSD is similar to Mode $1\left(I_{I M}=2.5 \mathrm{~A}\right)$ in Figure 10, but the cathode oscillations are not as pronounced as the H6MS. Local mode with a reference magnetic field $\left(B_{r} / B_{r}^{*}=1.00\right)$ in Figure. 14 exhibits a broad spectrum oscillation centered around $10 \mathrm{kHz}$ that is three orders of magnitude smaller than global mode oscillations. Sweep 3 shows a small peak at $70 \mathrm{kHz}$ that is approximately $1 / 4$ of the peak PSD value and 
may be related to cathode oscillations, but Sweep 1 and 2 do not show this feature. This PSD does not share the same qualitative features as Mode $2\left(I_{I M}=4.0 \mathrm{~A}\right)$ in Figure 10, where there are distinct peaks at 10 and $78 \mathrm{kHz}$. Local mode with a high magnetic field $\left(B_{r} / B_{r}^{*}=1.48\right)$ in Figure. 14 exhibits broad spectrum oscillations centered on $20 \mathrm{kHz}$ that are an order of magnitude larger than $B_{r} / B_{r}^{*}=1.00$, but cathode related oscillations are not readily apparent. This PSD has a broad low-frequency peak similar to Mode 3 $\left(I_{I M}=6.0 \mathrm{~A}\right)$ in Figure 10, but does not have the same broad peak in the range of cathode oscillations. Other magnetic field setting were investigated and the cathode type oscillations from $50-80 \mathrm{kHz}$ were not typically observed in local mode for the H6.

A time resolved plasma plume property map of the $\mathrm{H} 6$ in local mode $\left(B_{r} / B_{r}^{*}=1.00\right)$ is presented in Appendix B of Ref. 4, where cathode related oscillations from $68-83 \mathrm{kHz}$ are observed in the near-field plume region adjacent to the front pole. This indicates that plasma oscillations are occurring in the plume that do not typically couple strongly with the plasma in the discharge channel in order to affect the discharge current for non-magnetically shielded thrusters. The presence of these oscillations in the discharge current PSD of magnetically shielded thrusters and typical absence in unshielded thrusters operating in local mode suggests pushing the ionization zone further downstream and outside of the discharge channel causes a strong coupling between the near cathode plasma and discharge plasma.
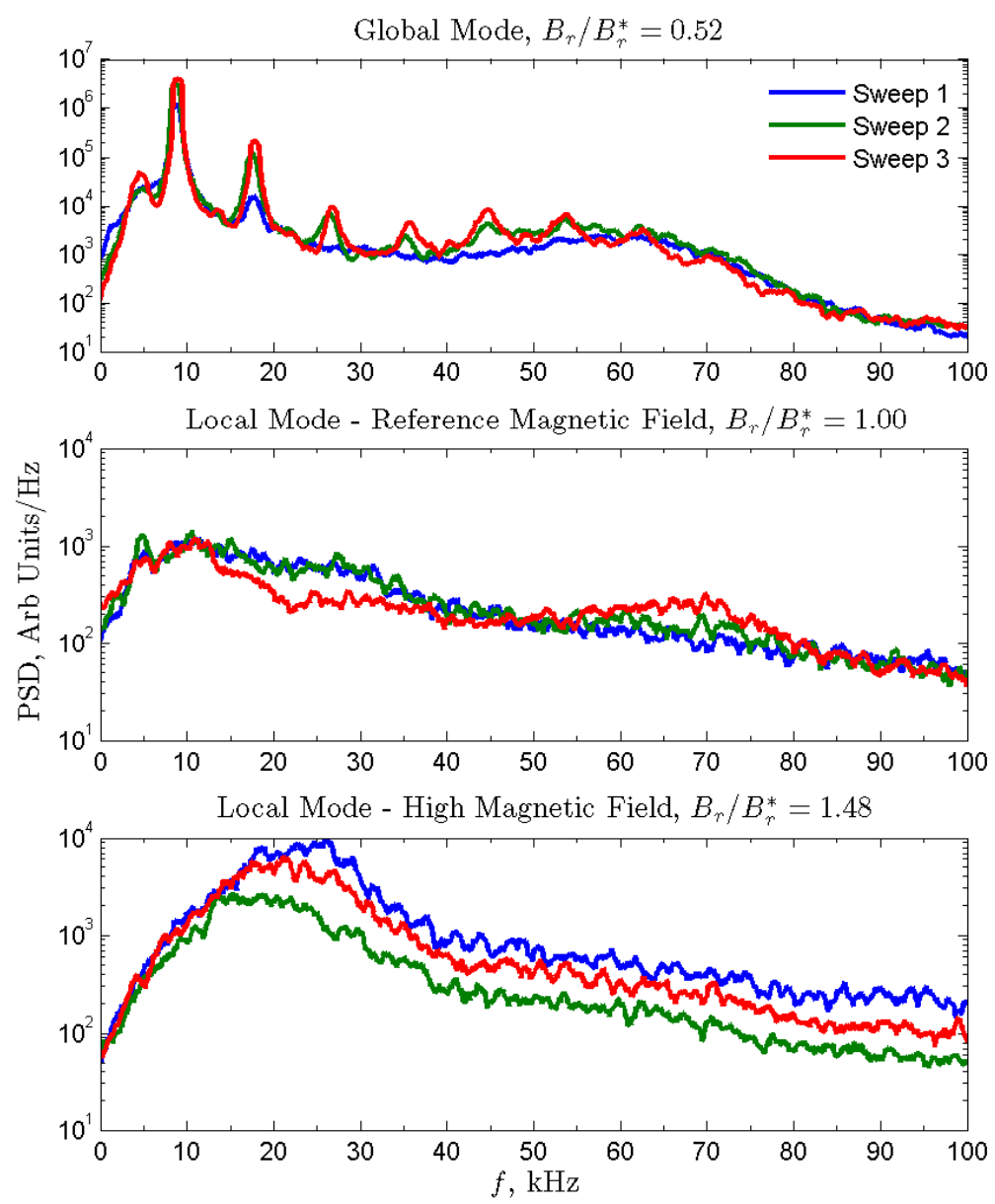

Figure 14. Discharge current PSDs for the non-magnetically shielded H6 at the $300 \mathrm{~V}, 19.5 \mathrm{mg} / \mathrm{s}$ condition during three different B-field sweeps. Three magnetic field settings are shown for global mode $\left(B_{r} / B_{r}^{*}=0.52\right)$, reference B-field local mode $\left(B_{r} / B_{r}^{*}=1.00\right)$, and high B-field local mode $\left(B_{r} / B_{r}^{*}=1.48\right)$. The values for $B_{r} / B_{r}^{*}$ are \pm 0.01 . The PSDs have been smoothed by a $1 \mathbf{~ k H z}$ wide moving average filter. The vertical scale for global mode is different than the two local mode conditions to accommodate the strong global mode oscillations. 


\section{Conclusions}

A mode transition study is conducted on magnetically shielded thrusters where the magnetic field magnitude is varied to induce mode transitions. Three different oscillatory modes are identified with the NASA300MS-2 and H6MS: Mode 1 at low magnetic fields, Mode 2 at nominal magnetic fields, and Mode 3 at high magnetic fields. The transition from Mode 1 to Mode 2 is similar to the global-to-local mode transition in unshielded thrusters, except that spokes are not observed in Mode 2, the equivalent of local mode. The equivalent global mode in magnetically shielded thrusters, Mode 1, exhibits large amplitude, low frequency $(1-10 \mathrm{kHz})$, breathing mode type oscillations. Mode 2 exhibits higher frequency $(50-90 \mathrm{kHz})$, low amplitude, entire channel oscillations that were previously identified as cathode related oscillations. This shows a strong coupling between the discharge plasma in magnetically shielded thrusters (which is further downstream compared to unshielded thrusters) and the cathode plasma. A second mode transition is observed in magnetically shielded thrusters at higher magnetic field magnitudes where spokes are observed simultaneously with cathode oscillations and breathing mode type oscillations in Mode 3. The spokes may be caused by off-nominal magnetic field topology resulting from a saturated magnetic circuit, which is under further investigation. The 300MS-2 $400 \mathrm{~V}$ condition exhibited a cathode oscillation resonance in Mode 2 close to the spoke transition that did not cause an increase in mean discharge current. The optimal thrust-to-power and anode efficiency are achieved in cathode oscillation mode or Mode 2. Thrust peaks in Mode 3 and decreases by $5-6 \%$ with decreasing magnetic field strength. The presence or absence of spokes and strong cathode oscillations do not affect each other or the discharge current. Similar to unshielded thrusters, mode transitions and plasma oscillations affect magnetically shielded thruster performance. As magnetically shielded thruster development continues, it will be important to understand and characterize the thruster oscillatory mode using time-resolved diagnostics.

\section{Acknowledgments}

The author (M. Sekerak) acknowledges support from a NASA Office of the Chief Technologist's Space Technology Research Fellowship (NSTRF). This work was also supported by AFOSR and AFRL through the MACEEP center of excellence grant number FA9550-09-1-0695. Support was also provided by subcontract RS140086 awarded by ERC, Inc. A portion of this research was carried out at the Jet Propulsion Laboratory, California Institute of Technology, under a contract with the National Aeronautics and Space Administration.

\section{References}

\footnotetext{
${ }^{1}$ Morozov, A. I., "The conceptual development of stationary plasma thrusters," Plasma Physics Reports, Vol. 29, No. 3, March 2003, pp. 235-250.

${ }^{2}$ Tilinin, G. N., "High-frequency plasma waves in a Hall accelerator with an extended acceleration zone," Soviet PhysicsTechnical Physics, Aug. 1977.

${ }^{3}$ Sekerak, M. J., Hofer, R. R., Polk, J. E., Longmier, B. W., Gallimore, A., and Brown, D. L., "Mode Transitions in Hall Effect Thrusters," AIAA 2013-4116, July 2013.

${ }^{4}$ Sekerak, M. J., Plasma Oscillations and Operational Modes in Hall Effect Thrusters, Ph.D. dissertation, University of Michigan, Ann Arbor, MI, 2014.

${ }^{5}$ Mikellides, I. G., Katz, I., Hofer, R. R., and Goebel, D. M., "Magnetic shielding of walls from the unmagnetized ion beam in a Hall thruster," Applied Physics Letters, Vol. 102, No. 2, 2013, pp. 023509.

${ }^{6}$ Hofer, R. R., Goebel, D. M., Mikellides, I. G., and Katz, I., "Magnetic shielding of a laboratory Hall thruster. II. Experiments," Journal of Applied Physics, Vol. 115, No. 4, Jan. 2014, pp. 043304.

${ }^{7}$ Mikellides, I. G., Katz, I., Hofer, R. R., Goebel, D. M., de Grys, K., and Mathers, A., "Magnetic shielding of the channel walls in a Hall plasma accelerator," Physics of Plasmas, Vol. 18, No. 3, 2011, pp. 033501.

${ }^{8}$ Mikellides, I. G., Katz, I., Hofer, R. R., Goebel, D. M., de Grys, K., and Mathers, A., "Magnetic Shielding of the Acceleration Channel Walls in a Long-Life Hall Thruster," AIAA-2010-6942, Nashville, TN, July 2010.

${ }^{9}$ Goebel, D. M., Hofer, R. R., Mikellides, I. G., Katz, I., Polk, J. E., and Dotson, B., "Conducting Wall Hall Thrusters," IEPC-2013-276, Washington, D.C., Oct. 2013.

${ }^{10}$ Gascon, N., Dudeck, M., and Barral, S., "Wall material effects in stationary plasma thrusters. I. Parametric studies of an SPT-100," Physics of Plasmas, Vol. 10, No. 10, 2003, pp. 4123.

${ }^{11}$ Brown, D. and Gallimore, A., "Investigation of Low Discharge Current Voltage Hall Thruster Operating Modes and Ionization Processes," IEPC-2009-074, Ann Arbor, MI, Sept. 2009.

${ }^{12}$ Sekerak, M. J., Longmier, B. W., Gallimore, A. D., Brown, D. L., Hofer, R. R., and Polk, J. E., "Azimuthal Spoke Propagation in Hall Effect Thrusters," IEPC-2013-143, Washington, D.C., Oct. 2013.
} 
${ }^{13}$ McDonald, M., Bellant, C., St Pierre, B., and Gallimore, A., "Measurement of Cross-Field Electron Current in a Hall Thruster Due to Rotating Spoke Instabilities," AIAA 2011-5810, San Diego, CA, Aug. 2011.

${ }^{14}$ Lobbia, R., Liu, T., and Gallimore, A., "Correlating time-resolved optical and Langmuir probe measurements of Hall thruster dynamics," SPS-III-36, Orlando, FL, Dec. 2008.

${ }^{15}$ Hara, K. and Boyd, I. D., "Low Frequency Oscillation Analysis of a Hall Thruster Using a One-Dimensional Hybrid-Direct Kinetic Simulation," IEPC-2013-266, Washington, D.C., Oct. 2013.

${ }^{16}$ McDonald, M. S. and Gallimore, A. D., "Comparison of Breathing and Spoke Mode Strength in the H6 Hall Thruster Using High Speed Imaging," IEPC-2013-353, Washington, D.C., Oct. 2013.

${ }^{17}$ Kamhawi, H., Haag, T., Jacobson, D., and Manzella, D., "Performance Evaluation of the NASA-300M 20 kW Hall Thruster," AIAA 2011-5521, San Diego, CA, July 2011.

${ }^{18}$ Huang, W., Shastry, R., Soulas, G. C., and Kamhawi, H., "Farfield Plume Measurements and Analysis on the NASA-300M and NASA-300MS," IEPC-2013-057, Washington, D.C., Oct. 2013.

${ }^{19}$ Haag, T. W., "Thrust stand for high-power electric propulsion devices," Review of Scientific Instruments, Vol. 62, No. 5, 1991, pp. 1186.

${ }^{20}$ Hofer, R. R., Development and characterization of high-efficiency, high-specific impulse xenon Hall thrusters., Ph.D. dissertation, 2004, $1 \mathrm{v}$.

${ }^{21}$ Hofer, R., Goebel, D., Mikellides, I., and Katz, I., "Design of a Laboratory Hall Thruster with Magnetically Shielded Channel Walls, Phase II: Experiments," AIAA 2012-3789, Atlanta, GA, Aug. 2012.

${ }^{22}$ Mikellides, I., Katz, I., and Hofer, R., "Design of a Laboratory Hall Thruster with Magnetically Shielded Channel Walls, Phase I: Numerical Simulations," AIAA 2011-5809, San Diego, CA, Aug. 2011.

${ }^{23}$ Mikellides, I., Katz, I., Hofer, R., and Goebel, D., "Design of a Laboratory Hall Thruster with Magnetically Shielded Channel Walls, Phase III: Comparison of Theory with Experiment," AIAA 2012-3789, Atlanta, GA, Aug. 2012.

${ }^{24}$ Jorns, B. A. and Hofer, R. R., "Plasma oscillations in a 6-kW magnetically shielded Hall thruster," Physics of Plasmas, Vol. 21, No. 5, May 2014, pp. 053512. 